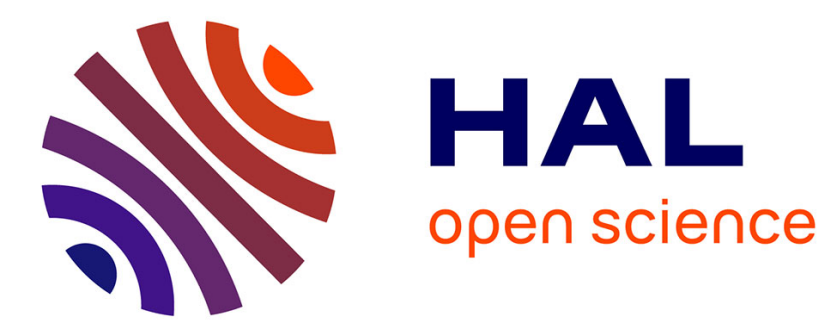

\title{
Low-Reynolds-number rising of a bubble near a free surface at vanishing Bond number
}

Marine Guemas, Antoine Sellier, Franck Pigeonneau

\section{To cite this version:}

Marine Guemas, Antoine Sellier, Franck Pigeonneau. Low-Reynolds-number rising of a bubble near a free surface at vanishing Bond number. Physics of Fluids, 2016. hal-01323532

\section{HAL Id: hal-01323532 \\ https://hal.science/hal-01323532}

Submitted on 30 May 2016

HAL is a multi-disciplinary open access archive for the deposit and dissemination of scientific research documents, whether they are published or not. The documents may come from teaching and research institutions in France or abroad, or from public or private research centers.
L'archive ouverte pluridisciplinaire HAL, est destinée au dépôt et à la diffusion de documents scientifiques de niveau recherche, publiés ou non, émanant des établissements d'enseignement et de recherche français ou étrangers, des laboratoires publics ou privés. 


\section{Low-Reynolds-number rising of a bubble near a free surface at vanishing Bond number}

Marine Guémas, ${ }^{1}$ Antoine Sellier, ${ }^{1}$ and Franck Pigeonneau ${ }^{2}$

1) Laboratoire d'Hydrodynamique (LadHyX), Ecole Polytechnique, 91128 Palaiseau cedex, France

2) Surface du Verre et Interfaces, UMR 125 CNRS/Saint-Gobain, 39 Quai Lucien Lefranc - BP 135, 93303 Aubervilliers cedex,

\section{France}

(Dated: 25 May 2016)

This work considers a nearly-spherical bubble and a nearly-flat free surface interacting under buoyancy at vanishing Bond number Bo. For each perturbed surface, the deviation from the unperturbed shape is asymptotically obtained at leading order on Bo. The task appeals to the normal traction exerted on the unperturbed surface by the Stokes flow due to a spherical bubble translating toward a flat free surface. The free surface problem is then found to be well-posed and to admit a solution in closed form when gravity is still present in the linear differential equation governing the perturbed profile through a term proportional to Bo. In contrast, the bubble problem amazingly turns out to be over-determined. It however becomes well-posed if the requirement of horizontal tangent planes at the perturbed bubble north and south poles is discarded or if the term proportional to Bo is omitted. Both previous approaches turn out to predict for small Bond number quite close solutions except in the very vicinity of the bubble poles. The numerical solution of the proposed asymptotic analysis shows, in the overlapping range $\mathrm{Bo}=\mathcal{O}(0.1)$ and for both the bubble and the free surface perturbed shapes, a good agreement with a quite different boundary element approach developed in [Phys. Fluids 23, 092102 (2011)]. It also provides approximated bubble and free surface shapes whose sensitivity to the bubble location is examined. 


\section{INTRODUCTION}

The motion of particles (solid bodies, bubbles or drops) rising toward a free surface or a liquid-liquid interface is encountered in many industrial applications such as flotation, distillation, liquid-liquid extraction. For close boundaries (particle surface, free surface or interface) strong interactions arise which not only dictate the non-solid particle(s) and interface shapes but also the liquid flow pattern. Hence, for most of the applications it is necessary to adequately handle such particle-boundary and/or particle-particle interactions. For instance, this is the case when estimating the lifetime of a bubble at a free surface, which is a key parameter to adequately predict the occurrence of foam in glass melting process ${ }^{1}$.

Once a particle (solid, drop, bubble) is finally stuck to an interface or a free surface (due to buoyancy) it lasts a long time before the rupture of the liquid film squeezed between the particle and this close surface. During this stage, the lubrication controls the film drainage. Actually, for small enough particles the liquid flows at low velocity not only during and but also before the drainage so that one can resort to the creeping flow assumption. Within this Low-Reynolds-Number flow simplified framework, Lee and Leal ${ }^{2}$ numerically investigated the axisymmetric slow rise of a solid sphere toward a liquid-liquid interface using a suitable boundary integral formulation. Simultaneously, Berdan and Leal ${ }^{3}$ addressed the same problem but for a sufficiently distant sphere and a weakly perturbed interface by working out an asymptotic analysis. Yiantsios and David ${ }^{4}$ later investigated the case of a solid sphere or drop approaching a deformable interface.

As already illustrated in the seminal contribution of Youngren and Acrivos ${ }^{5}$ for a solid body, the boundary element method (BEM) is a powerful technique to deal with steady Stokes flows. This well-established method (see textbooks such as ${ }^{6,7}$ and also for the numerical implementation ${ }^{8}$ ) is also quite efficient to track interacting non-rigid boundaries (free surface, interface) even for the case of fully three-dimensional liquid domains.

As experimentally evidenced for one bubble ${ }^{9}$, the free surface and bubble deformations

play a key role on the drainage dynamics. Therefore, it is necessary to accurately compute the shape of each encountered time-dependent interface. Unfortunately, this issue has been found by Pigeonneau and Sellier ${ }^{10}$ to be very challenging when the surface tension $\gamma$ becomes in a sense too large compared with the magnitude $g>0$ of the imposed uniform gravity field $\boldsymbol{g}$. More precisely, if the bubble(s) with a typical length $a$ are immersed in a liquid 
with uniform density $\rho_{l}$ and viscosity $\mu$, the numerical accuracy deteriorates as the Bond number $\mathrm{Bo}=\rho_{l} g a^{2} /(3 \gamma)$ vanishes. In practice, it is required to drastically refine the mesh on each interface and to decrease the time step as soon as Bo $\leq 0.2$ while for Bo $\leq 0.05$ numerical computations finally exhibit unphysical free surface and bubble shapes close the problem axis of revolution. Actually, this has to do with the accuracy level at which one approximates on the entire liquid boundary $S$ both the unit normal $\boldsymbol{n}$ and the curvature given by the surface divergence of $\boldsymbol{n}: \nabla_{S} \cdot \boldsymbol{n}$. Indeed, if the computational error made on $a \nabla_{S} \cdot \boldsymbol{n}$ is order $\epsilon_{a}>0$ the resulting numerical error for the capillary force is order $\epsilon_{a} /$ Bo and thus becomes too large when Bo vanishes. This error then worsens the determination of the flow velocity $\boldsymbol{u}$ on the entire surface $S$ and therefore the liquid domain boundary $S$ time-dependent location ${ }^{10}$. However, at small Bo each bubble and the free surface remain nearly-spherical and nearly-flat, respectively and this suggests gaining the weakly perturbed shapes by developing an asymptotic analysis in terms of the small Bond number Bo.

The aim of the present work is to asymptotically approximate at leading order in small Bond number the shapes of two interacting and gravity-driven bubble and free surface (i.e. to treat for only one bubble the problem encountered by Pigeonneau and Sellier ${ }^{10}$ for vanishing Bo). Similar issues have been actually addressed in the literature but for different circumstances. One can first cite the case of a bubble or droplet with large uniform surface tension $\gamma$ and subject (in absence of gravity) to an arbitrary ambient steady Stokes flow with velocity magnitude $V$. This problem has been nicely handled by Hetsroni and Haber ${ }^{11}$ in which the nearly-spherical particle shape is approximated at the first order in Capillary number $\mathrm{Ca}=\mu V / \gamma$ whatever the ambient Stokes flow. As earlier mentioned, Berdan and $\mathrm{Leal}^{3}$ later asymptotically obtained the nearly-flat shape of a fluid-fluid interface interacting with a solid and not-necessarily force-free sphere with radius $a$ as both capillary number Ca and Bond number Bo vanish. Finally, one should mention two additional papers dealing with two nearly spherical and weakly interacting drops moving either in the same liquid in absence of gravity ${ }^{12}$ or in two different liquids due to the gravity ${ }^{13}$ (with in this latter case also the approximation of the nearly-flat liquid-liquid interface). One should note that the results obtained by Chervenivanova and Zapryanov ${ }^{13}$ amazingly predict, a non-smooth perturbed interface and are therefore questionable. In addition, there is to the authors very best knowledge no work dealing with the case of a bubble interacting with a free surface at small Bond number. 


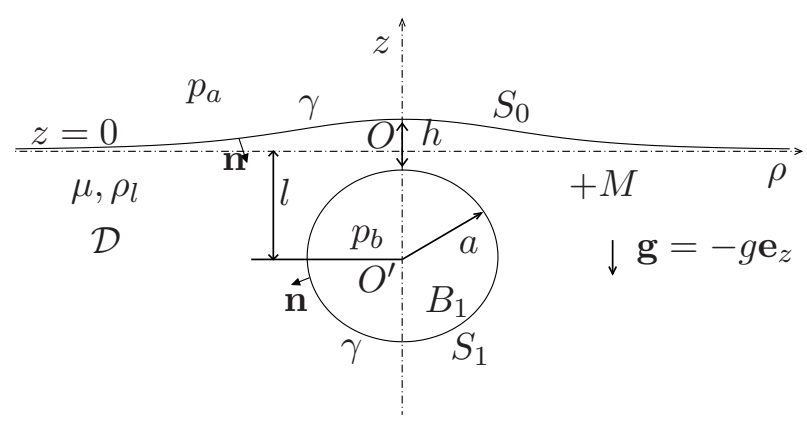

FIG. 1: A nearly-spherical bubble $B_{1}$ ascending, under the uniform gravity field $\boldsymbol{g}=-g \boldsymbol{e}_{z}$, toward a weakly perturbed free surface $S_{0}$.

The paper is organized as follows. The governing Stokes problem and its associated zeroth-order flow are presented in $\S I I$. The free-surface location first-order approximation in small capillary number is obtained in $\S$ III while $\S$ IV is devoted to the estimate, at the same order, of the weakly non-spherical bubble shape. The proposed asymptotic theory is compared in $\S \mathrm{V}$ with the BEM predictions provided by Pigeonneau and Sellier ${ }^{10}$ while concluding remarks close the paper in $\S$ VI.

\section{GOVERNING PROBLEM AND ZEROTH-ORDER FLOW SOLUTION}

This section presents the governing axisymmetric Stokes problem and the flow asymptotic expansion at relevant vanishing Bond and capillary numbers.

\section{A. Axisymmetric Stokes problem}

As sketched in FIG. 1, we consider a bubble ascending, in a Newtonian liquid with a uniform density $\rho_{l}$, toward a free surface under a uniform gravity field $\boldsymbol{g}=-g \boldsymbol{e}_{z}$. For distant bubble and free surface this bubble is spherical with a radius $a$ while the free surface is the $z=0$ plane. Otherwise, at time $t$ the non-spherical bubble center-of-volume $O^{\prime}$ is located at a distance $l$ from the $z=0$ plane. In the liquid domain $\mathcal{D}(t)$ the fluid has a velocity field $\boldsymbol{u}$, with a magnitude $U$, and a pressure field $p+\rho_{l} \boldsymbol{g} \cdot \boldsymbol{x}+p_{a}$ where $\boldsymbol{x}=\mathbf{O M}$ and $p_{a}$ designates the uniform ambient pressure above the free surface. Assuming a vanishing Reynolds number, i.e. $\operatorname{Re}=\rho_{l} U a / \mu \ll 1$, and a flow quiescent far from the bubble it follows 
that $(\boldsymbol{u}, p)$ obeys

$$
\begin{aligned}
& \mu \nabla^{2} \boldsymbol{u}=\nabla p \text { and } \nabla \cdot \boldsymbol{u}=0 \text { in } \mathcal{D}(t) \\
& (\boldsymbol{u}, p) \rightarrow(\mathbf{0}, 0) \text { as }|\boldsymbol{x}| \rightarrow \infty
\end{aligned}
$$

We supplement (1)-(2) with boundary conditions on the free surface $S_{0}$ and the bubble boundary $S_{1}$ having a unit outward normal $\boldsymbol{n}$ and a local average curvature $H=\nabla_{S} \cdot \boldsymbol{n} / 2$ with $\nabla_{S}$ the surface divergence ${ }^{14}$. Assuming the same uniform surface tension $\gamma$ on each surface and denoting by $p_{b}$ the bubble uniform pressure and by $\boldsymbol{\sigma}$ the flow $(\boldsymbol{u}, p)$ stress tensor yields $^{15}$

$$
\begin{aligned}
& \boldsymbol{n} \cdot \boldsymbol{\sigma} \cdot \boldsymbol{n}=\rho_{l} \boldsymbol{g} \cdot \boldsymbol{x}+\gamma \boldsymbol{\nabla}_{S} \cdot \boldsymbol{n} \text { on } S_{0}, \\
& \boldsymbol{n} \cdot \boldsymbol{\sigma} \cdot \boldsymbol{n}=\rho_{l} \boldsymbol{g} \cdot \boldsymbol{x}+p_{a}-p_{b}+\gamma \nabla_{S} \cdot \boldsymbol{n} \text { on } S_{1}, \\
& (\boldsymbol{\sigma} \cdot \boldsymbol{n}) \wedge \boldsymbol{n}=\mathbf{0} \text { on } S_{0}, S_{1} .
\end{aligned}
$$

There is no mass transfer across $S_{0} \cup S_{1}$ and the bubble has a constant volume $\mathcal{V}_{b}$. Thus,

$$
\int_{S_{1}} \boldsymbol{u} \cdot \boldsymbol{n} d S=0
$$

At each time $t$ one gains $(\boldsymbol{u}, p)$ by solving the well-posed problem (1)-(6). Note that:

(i) The bubble is force free since integrating (4) over $S_{1}$ with $p_{a}-p_{b}$ uniform gives a zero contribution $^{16}$.

(ii) To track in time the liquid boundary $S_{0} \cup S_{1}$, with the material velocity $\boldsymbol{U}$, it is sufficient to first get there the velocity $\boldsymbol{u}$ and then to exploit the no-mass transfer condition

$$
\boldsymbol{U} \cdot \boldsymbol{n}=\boldsymbol{u} \cdot \boldsymbol{n} \text { on } S_{0} \cup S_{1}
$$

\section{B. Dimensionless numbers and flow expansion at vanishing Bond number}

In (3)-(4) the terms $\boldsymbol{n} \cdot \boldsymbol{\sigma} \cdot \boldsymbol{n}, \rho_{l} \boldsymbol{g} \cdot \boldsymbol{x}$ and $\gamma \boldsymbol{\nabla}_{S} \cdot \boldsymbol{n}$ are of flow, gravity and capillary natures. Taking $U$ as typical velocity magnitude and the bubble initial radius $a$ as length scale give

$$
\gamma \nabla_{S} \cdot \boldsymbol{n} \sim \gamma / a, \quad \boldsymbol{n} \cdot \boldsymbol{\sigma} \cdot \boldsymbol{n} \sim \mu U / a, \quad \rho_{l} \boldsymbol{g} \cdot \boldsymbol{x} \sim \rho_{l} g a \text { on } S_{0} \cup S_{1}
$$

Comparing either in (3) or in (4) the gravity term and the flow term with the capillary term then introduces two dimensionless numbers: the Bond number Bo and the capillary number Ca here defined as

$$
\mathrm{Bo}=\frac{\rho_{l} g a^{2}}{3 \gamma}, \quad \mathrm{Ca}=\frac{\mu U}{\gamma} .
$$




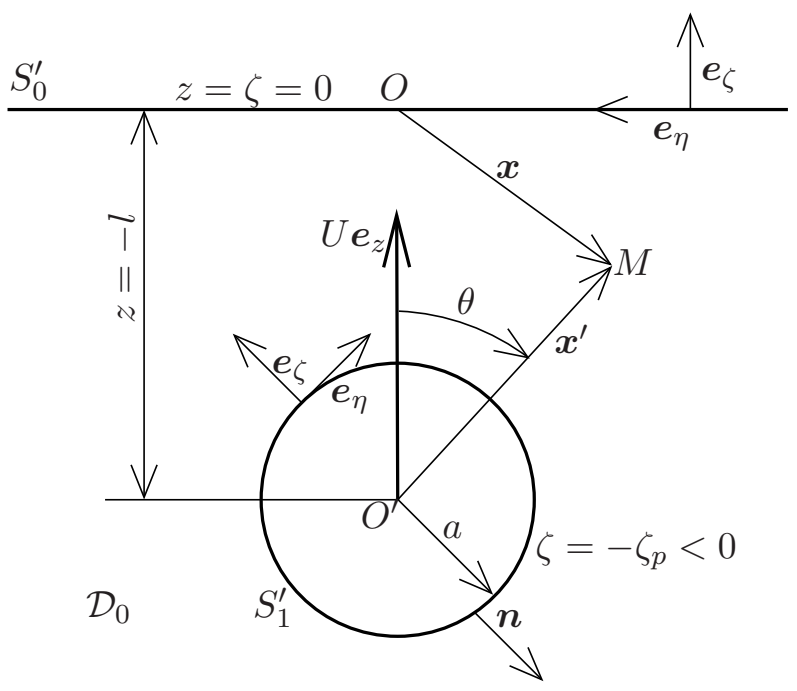

FIG. 2: Zeroth-order axisymmetric problem of a spherical bubble with radius $a$, center $O^{\prime}$ and surface $S_{1}^{\prime}$ translating at the velocity $U \boldsymbol{e}_{z}$ toward the $z=0$ flat free surface $S_{0}^{\prime}$.

For the present problem $\mathrm{Ca}=\mathcal{O}(\mathrm{Bo})$ since, due to the cancellation of the integral of $(4)$, $\mu U a \sim \rho_{l} g a^{3}$.

Henceforth, it is assumed that $\mathrm{Bo} \ll 1$ and therefore also that $\mathrm{Ca} \ll 1$. In that case the bubble and the free surface remain nearly spherical and flat, respectively while the flow $(\boldsymbol{u}, p)$ is expanded as follows

$$
\boldsymbol{u}=\boldsymbol{u}_{0}+\mathrm{Ca} \boldsymbol{u}_{1}+\mathcal{O}\left(\mathrm{Ca}^{2}\right), p=p_{0}+\mathrm{Ca} p_{1}+\mathcal{O}\left(\mathrm{Ca}^{2}\right)
$$

The zeroth-order flow $\left(\boldsymbol{u}_{0}, p_{0}\right)$, with stress tensor $\boldsymbol{\sigma}_{0}$, is handled in $\S$ II C while $\left(\boldsymbol{u}_{1}, p_{1}\right)$ is discarded because, as shown in sections $\S$ III and $\S \mathrm{IV}$, determining the first-order $\mathcal{O}(\mathrm{Ca})$ weakly perturbed free surface and bubble shapes solely appeals to the normal stress $\boldsymbol{n}_{0} \cdot \boldsymbol{\sigma}_{0} \cdot \boldsymbol{n}_{0}$ on the unperturbed spherical bubble surface and flat free surface with the unit normal $\boldsymbol{n}_{0}$.

\section{Zeroth-order flow problem and solution}

\section{Zeroth-order flow and drag coefficient}

As illustrated in FIG. 2, $\left(\boldsymbol{u}_{0}, p_{0}\right)$ is the flow about a spherical bubble with a radius $a$ and surface $S_{1}^{\prime}$ translating at the velocity $\boldsymbol{u}_{0}=U \boldsymbol{e}_{z}$ toward $(U>0)$ the motionless $z=0$ plane $S_{0}^{\prime}$. The liquid domain is $\mathcal{D}_{0}$ and $\left(\boldsymbol{u}_{0}, p_{0}\right)$ obeys, using $(7)$, 


$$
\begin{aligned}
& \mu \boldsymbol{\nabla}^{2} \boldsymbol{u}_{0}=\nabla p_{0} \text { and } \boldsymbol{\nabla} \cdot \boldsymbol{u}_{0}=0 \text { in } \mathcal{D}_{0}, \quad\left(\boldsymbol{u}_{0}, p_{0}\right) \rightarrow(\mathbf{0}, 0) \text { as }|\boldsymbol{x}| \rightarrow \infty, \\
& \boldsymbol{u}_{0} \cdot \boldsymbol{n}_{0}=0 \text { on } S_{0}^{\prime}, \quad \boldsymbol{u}_{0} \cdot \boldsymbol{n}_{0}=U \boldsymbol{e}_{z} \cdot \boldsymbol{n}_{0} \quad \text { on } S_{1}^{\prime}, \quad\left(\boldsymbol{\sigma}_{0} \cdot \boldsymbol{n}_{0}\right) \wedge \boldsymbol{n}_{0}=\mathbf{0} \text { on } S_{0}^{\prime} \cup S_{1}^{\prime} .
\end{aligned}
$$

For symmetry reasons, $\left(\boldsymbol{u}_{0}, p_{0}\right)$ exerts on the bubble a zero torque and a force $\boldsymbol{F}_{0}$ given by

$$
\boldsymbol{F}_{0}=\int_{S_{1}^{\prime}} \boldsymbol{\sigma}_{0} \cdot \boldsymbol{n} d S=-4 \pi \mu U a \lambda_{0} \boldsymbol{e}_{z}
$$

with drag coefficient $\lambda_{0}>1$ (see Table I in $\S$ II C 2) solely depending upon the bubble normalized $l / a$. For the freely-suspended bubble, expanding (B5) at the leading order in small Bo yields

$$
U=\frac{\rho_{l} g a^{2}}{3 \mu \lambda_{0}}, \lambda_{0}=\mathrm{Bo} / \mathrm{Ca}=\mathcal{O}(1)
$$

\section{Solution in bipolar coordinates and comparisons}

As in ${ }^{17,18}$, the axisymmetric problem (11)-(12) is solved using the usual bipolar coordinates $(\zeta, \eta, \phi)$ here related to the cylindrical coordinates $(\rho, z, \phi)$ as follows ${ }^{19}$

$$
z=\frac{c \sinh \zeta}{\cosh \zeta-\cos \eta}, \rho=\frac{c \sin \eta}{\cosh \zeta-\cos \eta}, c=\sqrt{l^{2}-a^{2}}
$$

Surfaces $\zeta=0$ and $\zeta=-\zeta_{p}$ for $\sinh \zeta_{p}=c / a$ are the boundaries $S_{0}^{\prime}$ and $S_{1}^{\prime}$, respectively. Local unit normal vectors $\boldsymbol{e}_{\zeta}, \boldsymbol{e}_{\eta}, \boldsymbol{e}_{\phi}=\boldsymbol{e}_{\eta} \wedge \boldsymbol{e}_{\zeta}$ are introduced at each point $\boldsymbol{x}(\zeta, \eta, \phi)$ in the liquid domain $-\zeta_{p} \leq \zeta \leq 0, \eta \in[0, \pi]$ and $\phi \in[0,2 \pi]$ (see FIG. 2). Setting $\chi=\cos \eta$, one gets $\boldsymbol{u}_{0}(\boldsymbol{x})=u_{0 \zeta} \boldsymbol{e}_{\zeta}+u_{0 \eta} \boldsymbol{e}_{\eta}$ with

$$
u_{0 \zeta}(\zeta, \chi)=-\frac{(\cosh (\zeta)-\chi)^{2}}{c^{2}} \frac{\partial \psi}{\partial \chi}, u_{0 \eta}(\zeta, \chi)=-\frac{(\cosh (\zeta)-\chi)^{2}}{c^{2} \sin (\eta)} \frac{\partial \psi}{\partial \zeta}
$$

and a stream function $\psi(\zeta, \chi)$ recalled $^{20}$ in Appendix A. On $S_{0}^{\prime}$ and $S_{1}^{\prime}$ the required normal stress reads $\boldsymbol{n}_{0} \cdot \boldsymbol{\sigma}_{0} \cdot \boldsymbol{n}_{0}=\sigma_{0 \zeta \zeta}=-p_{0}+\tau_{0 \zeta \zeta}$ with $p_{0}$ the pressure obtained as detailed in ${ }^{21}$ and $\tau_{0 \zeta \zeta}$ the normal viscous stress given by ${ }^{19}$

$$
\tau_{0 \zeta \zeta}(\zeta, \chi)=-2 \frac{\mu U(\cosh \zeta-\chi)}{c^{3}}\left\{\frac{\partial}{\partial \zeta}\left[(\cosh \zeta-\chi)^{2} \frac{\partial \psi}{\partial \chi}\right]-(\cosh \zeta-\chi) \frac{\partial \psi}{\partial \zeta}\right\} .
$$

The resulting values of $\sigma_{0 \zeta \zeta}$ on both $S_{0}^{\prime}(\zeta=0)$ and $S_{1}^{\prime}\left(\zeta=-\zeta_{p}\right)$, available $i^{22}$, are here tested in TABLE I by integrating $\sigma_{0 \zeta \zeta}$ (as detailed in Appendix A) on those surfaces to calculate the drag coefficient $\lambda_{0}$ introduced by (13). Clearly, there is an excellent agreement between the different approaches. 


\begin{tabular}{cccccc}
\hline$\left(\zeta_{p}, N_{t}\right)$ & $l / a$ & $\lambda_{0, a}{ }^{17}$ & $\lambda_{0, a}$ & $\lambda_{0,1}$ & $\lambda_{0,0}$ \\
\hline$(0.5,35)$ & $\sim 1.13$ & 2.049981 & 2.04998112191844 & 2.04998112191524 & 2.04998112205233 \\
$(0.5,50)$ & $\sim 1.13$ & 2.049981 & 2.04998112191844 & 2.04998112191845 & 2.04998112192156 \\
$(1,18)$ & $\sim 1.54$ & 1.509456 & 1.50945547483087 & 1.50945547482972 & 1.50945547488896 \\
$(1,25)$ & $\sim 1.54$ & 1.509456 & 1.50945547483087 & 1.50945547483086 & 1.50945547483031 \\
$(1.5,15)$ & $\sim 2.35$ & 1.273450 & 1.27344998699464 & 1.27344998699464 & 1.27344998699378 \\
\hline
\end{tabular}

TABLE I: Computed drag coefficients versus the bubble location $\zeta_{p}$ and the truncation number $N_{t}$ (see Appendix A) by analytical evaluation $\left(\lambda_{0, a}\right)$, integration over $S_{1}^{\prime}\left(\lambda_{0,1}\right)$ or integration over $S_{0}^{\prime}\left(\lambda_{0,0}\right)$. The 6 -digit value of $\lambda_{0, a}$ obtained by Bart ${ }^{17}$ is given for comparison.

\section{FIRST-ORDER WEAKLY PERTURBED FREE SURFACE SHAPE}

\section{A. Governing problem for the free surface shape}

The free surface shape location is obtained from (3) which gives the normal traction $\boldsymbol{n} \cdot \boldsymbol{\sigma} \cdot \boldsymbol{n}$ on the perturbed free surface $S_{0}$. Setting $d=2 a$, we adopt dimensionless quantities $\bar{z}=z / d, \bar{\rho}=\rho / d, \bar{\nabla}_{S} \cdot \boldsymbol{n}=d \boldsymbol{\nabla}_{S} \cdot \boldsymbol{n}$ and $\overline{\boldsymbol{\sigma}}=d \boldsymbol{\sigma} /(\mu U)$. The perturbed free surface $S_{0}$ admits equation $\bar{z}=\operatorname{Ca} f(\bar{\rho})$ with $f=\mathcal{O}(1)$ the unknown shape function. Moreover, it has unit normal $\boldsymbol{n} \sim-\boldsymbol{e}_{z}+\mathrm{Ca} \boldsymbol{n}_{1}$ with $^{3}$

$$
\left[\bar{\nabla}_{S} \cdot \boldsymbol{n}_{1}\right](f)=\frac{d^{2} f}{d \bar{\rho}^{2}}+\frac{1}{\bar{\rho}} \frac{d f}{d \bar{\rho}} .
$$

Recalling that $\boldsymbol{g}=-g \boldsymbol{e}_{z}$, substituting $\boldsymbol{n}$ and $\bar{z}$ in (3) and retaining the leading order terms yields, in conjunction with (18), the governing equation for the shape function

$$
\bar{\rho} \frac{d^{2} f}{d \bar{\rho}^{2}}+\frac{d f}{d \bar{\rho}}-t_{f} \bar{\rho} f=\bar{\rho}\left[\boldsymbol{n}_{0} \cdot \overline{\boldsymbol{\sigma}}_{0} \cdot \boldsymbol{n}_{0}\right](z=0) \text { with } t_{f}=12 \text { Bo }
$$

The free surface is unperturbed far from the $\left(z^{\prime} O z\right)$ axis and exhibits (axisymmetric problem) an horizontal tangent on the $\left(z^{\prime} O z\right)$ axis. Therefore, we supplement (19) with the conditions

$$
\frac{d f}{d \bar{\rho}}=0 \text { for } \bar{\rho}=0, f(\bar{\rho})=0 \text { as } \bar{\rho} \rightarrow \infty .
$$




\section{B. Basic property and analytical solution}

In solving (19)-(20), one may think about neglecting the term $t_{f} \bar{\rho} f$ in (19) because $\mathrm{Bo}=\mathcal{O}(\mathrm{Ca})$ for the freely suspended bubble. Doing so, and taking into account of the first condition (20) would give

$$
\frac{d f}{d \bar{\rho}}=\frac{g(\bar{\rho})}{\bar{\rho}}, f(\bar{\rho})=\int_{\infty}^{\bar{\rho}} \frac{g(s)}{s} d s, g(s)=\int_{0}^{s} u\left[\boldsymbol{n}_{0} \cdot \overline{\boldsymbol{\sigma}}_{0} \cdot \boldsymbol{n}_{0}\right](u) d u .
$$

But from (A6) one gains $g(s) \rightarrow-\lambda_{0} \neq 0$ as $s$ becomes large and thus $f$ given by (21) is not bounded! Thus, one must keep the term $t_{f} \bar{\rho} f$ in (19). Moreover, for a freely-suspended bubble the volume of liquid above the $z=0$ plane does not depend upon $(l / a, \mathrm{Bo})$ and is

equal to the bubble volume. Indeed, when normalized by $8 a^{3}$, this volume $\overline{\mathcal{V}}_{l}$ satisfies

$$
\overline{\mathcal{V}}_{l}=2 \pi \int_{0}^{\infty} \operatorname{Ca} f(\bar{\rho}) \bar{\rho} d \bar{\rho}=-\frac{\pi}{6 \lambda_{0}} \int_{0}^{\infty} \bar{\sigma}_{0 \zeta \zeta}(u) u d u=\frac{\pi}{6} .
$$

This is due to (19) which with $\lambda_{0}=\mathrm{Bo} / \mathrm{Ca}$ for the freely-suspended bubble becomes

$$
\frac{d}{d \bar{\rho}}\left(\bar{\rho} \frac{d f}{d \bar{\rho}}\right)=12 \lambda_{0} \operatorname{Ca} \bar{\rho} f(\bar{\rho})+\bar{\rho} \bar{\sigma}_{0 \zeta \zeta} .
$$

Anticipating on (24) it is possible to show that $\bar{\rho} d f / d \bar{\rho} \rightarrow 0$ as $\bar{\rho} \rightarrow \infty$. This latter property and the boundary condition $d f / d \bar{\rho}=0$ at $\bar{\rho}=0$ (see (20)) then provide (22) by integrating (23) over $[0, \infty[$.

Using the so-called method of Wronskian as done by Berdan and $\mathrm{Leal}^{3}$ for a distant solid sphere, provides the following analytical solution to (19)-(20)

$$
f(\bar{\rho})=I_{0}\left(\sqrt{t_{f}} \bar{\rho}\right) \int_{\bar{\rho}}^{\infty} K_{0}\left(\sqrt{t_{f}} u\right), u \bar{\sigma}_{0 \zeta \zeta}(u) d u-K_{0}\left(\sqrt{t_{f}} \bar{\rho}\right) \int_{0}^{\bar{\rho}} I_{0}\left(\sqrt{t_{f}} u\right) u \bar{\sigma}_{0 \zeta \zeta}(u) d u
$$

where $\bar{\sigma}_{0 \zeta \zeta}=\boldsymbol{n}_{0} \cdot \overline{\boldsymbol{\sigma}}_{0} \cdot \boldsymbol{n}_{0}=2 a \sigma_{0 \zeta \zeta} /(\mu U)$ and $I_{0}$ or $K_{0}$ denotes the usual modified Bessel functions of the first or second kind, respectively ${ }^{23}$. From $(24)$ and $I_{0}(0)=1$ it appears that the free surface shape function on the $\left(z^{\prime} O z\right)$ takes the value

$$
f(0)=\int_{0}^{\infty} K_{0}\left(\sqrt{t_{f}} u\right) u \bar{\sigma}_{0 \zeta \zeta}(u) d u
$$

\section{Numerical results and discussion}

The shape function $f$ is computed from (24) with a Fortran routine using the Netlib Library for the modified Bessel functions $I_{0}$ and $K_{0}$. 
To our very best knowledge no asymptotic result for a bubble is available in the literature and we thus first compare our results with the predictions of Berdan and Leal ${ }^{3}$ for a distant solid sphere. For a distant particle (solid sphere or bubble) translating at the velocity $U \boldsymbol{e}_{z}$ the free surface is, at the leading order, pushed by the flow produced by a point force located at the particle center of volume. This point force has strength $\boldsymbol{F}_{0}=-4 \pi \mu U a \lambda_{0} \boldsymbol{e}_{z}$ for the bubble and strength $\boldsymbol{F}_{0, s}=-6 \pi \mu U a \lambda_{0, s} \boldsymbol{e}_{z}$ for the solid sphere with drag coefficient $\lambda_{0, s}$ given in Bart ${ }^{17}$ using bipolar coordinates. For prescribed $(l / a, \mathrm{Bo}, \mathrm{Ca})$ the free surface shapes $f$ and $f_{s}$ obtained for distant bubble and solid sphere then obey $f_{s} / f=\left\|\boldsymbol{F}_{0, s}|/|\right\| \boldsymbol{F}_{0} \|=$ $3 \lambda_{0, s} /\left(2 \lambda_{0}\right)$. This relation is tested for $l / a=6, \mathrm{Bo}=1 / 3$ and $\mathrm{Ca}=0.2$ by plotting in FIG. 3 the free surface locations $z / a$ obtained for shape functions $f_{b}$ given by $(24), f_{s}=$ $3 \lambda_{0, s} f_{b} /\left(2 \lambda_{0}\right) \sim 1.570 f_{b}{ }^{24}, f_{\text {bel }}$ given for a solid sphere in $^{3}$ and finally $f_{s b i p}$ or $f_{s a}$ obtained by using in (24) the normal stress $\bar{\sigma}_{0 \zeta \zeta}(u)$ for a solid sphere either calculated in bipolar coordinates as in Bart ${ }^{17}$ for $f_{\text {sbip }}$ or asymptotically evaluated as in Berdan and Leal ${ }^{3}$ for $f_{s a}$. Clearly, the results for the distant solid sphere using $f_{s}, f_{b e l}, f_{s b i p}$ and $f_{s a}$ are consistent and predict a more deformed free surface than for the case of a bubble (using $f_{b}$ ) because of different (no-slip or no tangential stress) boundary conditions on the particle (sphere, bubble) surface.

We now examine the free surface deformation due to the freely-suspended bubble by prescribing the Bond number $\mathrm{Bo}$ and taking $\mathrm{Ca}=\mathrm{Bo} / \lambda_{0}$ with $\lambda_{0}$ given by (A4). In FIG. 4 we plot $z / a=2 \mathrm{Ca} f$ versus $\rho / a$ still for $l / a=6$ but at different Bo. Since $\lambda_{0} \sim 1.091$ for $l=6 a$ one has $\mathrm{Ca} \sim 0.3055$ at $\mathrm{Bo}=1 / 3$ in FIG. 4 . From $z / a=2 \mathrm{Ca} f(\rho / a ; l / a$, Bo $)$ one gets $(z / a)_{\text {FIG. } 4} \sim 1.528(z / a)_{\text {FIG.3 }}$ while for FIG. 3 recall that $f_{s} \sim 1.570 f_{b}$. This explains why curves for $z / a$ in FIG. 3 for the solid sphere and in FIG. 4 at Bo $=1 / 3$ are very close. As seen in FIG. 4, increasing Bo (by dropping the free surface tension at prescribed gravity) increases the free surface deformation near the $\left(z^{\prime} O z\right)$ axis. As shown in FIG. 5(a), this trend holds whatever the bubble location $l / a$. In contrast, the free surface sensitivity to $(l / a, \mathrm{Bo})$ far away from the $\left(z^{\prime} O z\right)$ axis is less intuitive. This is already seen for $l / a=6$ in FIG. 4 when $\rho / a \geq 5.5$, and confirmed by plotting in FIG. 5(b) the free surface deformation $z / a$ at $\rho / a=10$ versus $(l / a, \mathrm{Bo})$. At given $l / a$ this deformation increases as Bo drops from $1 / 3$ to 0.05 while it increases with Bo in the range [0.005, 0.01]. Moreover, depending on $l / a$, the value of $z / a$ at $\mathrm{Bo}=0.05$ is either larger or smaller than its value at $\mathrm{Bo}=0.005$. At $\rho / a=10$ this free surface deformation $z / a$ amazing sensitivity to Bo is due to the volume 


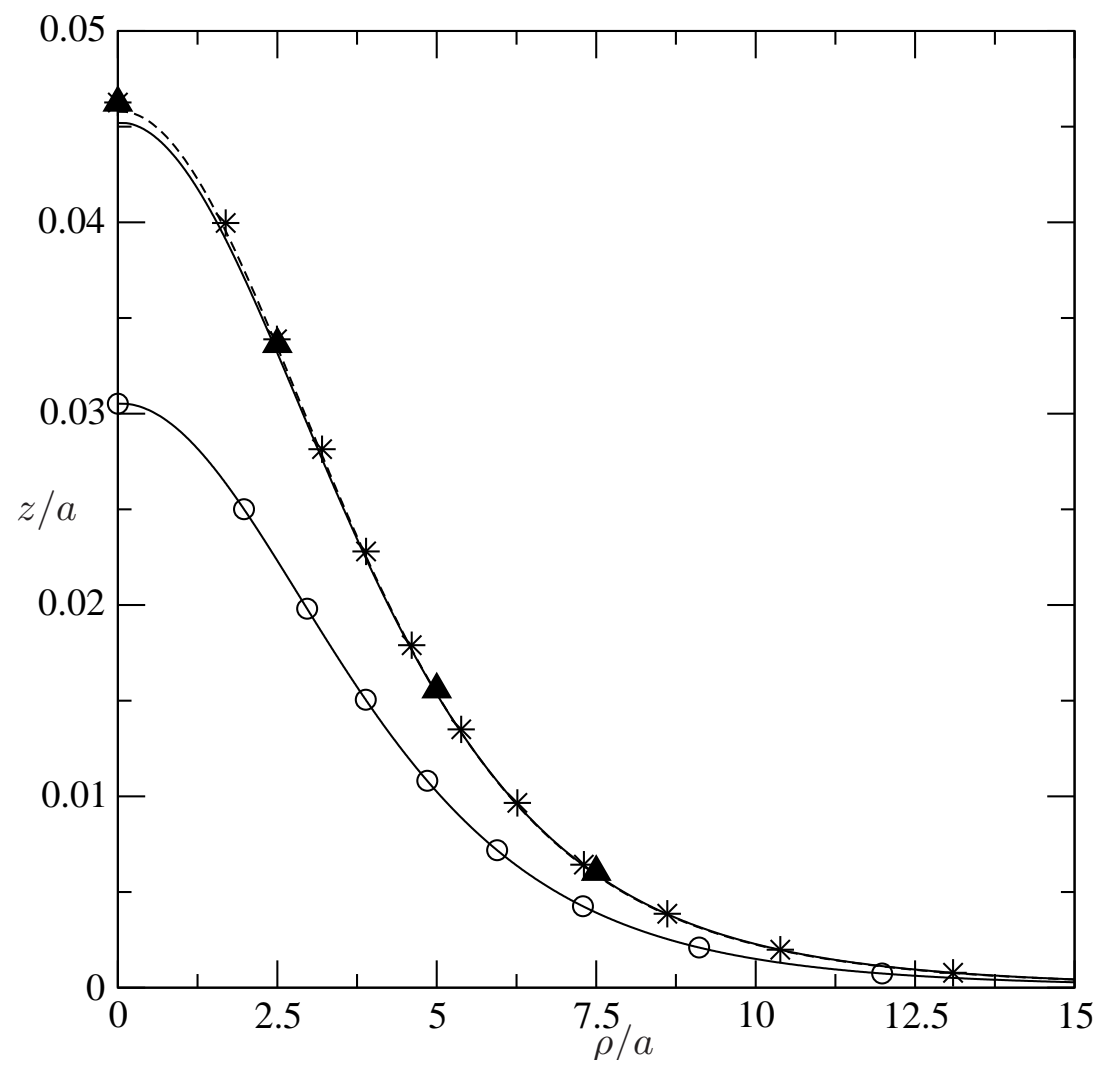

FIG. 3: Free surface shape locations $z / a=2$ Ca $f$ versus $\rho / a$ induced by distant bubble and solid sphere located at $l=6 a$ for $\mathrm{Bo}=1 / 3$ and $\mathrm{Ca}=0.2 . f_{b}(\circ), f_{s}$ (dashed line), $f_{\text {bel }}(*), f_{\text {sbip }}$ (solid curve) and $f_{\text {sa }}(\mathbf{\Delta})$.

conservation (22). Finally, FIG. 5 also shows that when $l / a$ drops at given Bo then $z / a$ increases at $\rho=0$ but, depending on Bo, either increases or decreases at $\rho=10 a$. The computed free surface locations for $l / a=4,2$ are displayed in FIG. 6 and exhibit the same trend as the ones discussed for FIG. 4. The developed first-order asymptotic analysis writes the free surface location as $z=2 a$ Ca $f$ with $f=\mathcal{O}(1)$. This latter assumption is satisfied as shown by plotting in FIG. 7(a) the normalized free surface location $z /(a \mathrm{Ca})$ at $\rho=0$ (i.e. where $f$ reaches its largest value) versus $l / a$ for several values of Bo. Not surprisingly, as the bubble approaches $2 f(0)$ increases but remains of order unity. The ratio $z /(a$ Bo $)$, plotted in FIG. 7(b), exhibits the same behaviour as the one observed in FIG. 7(a). 


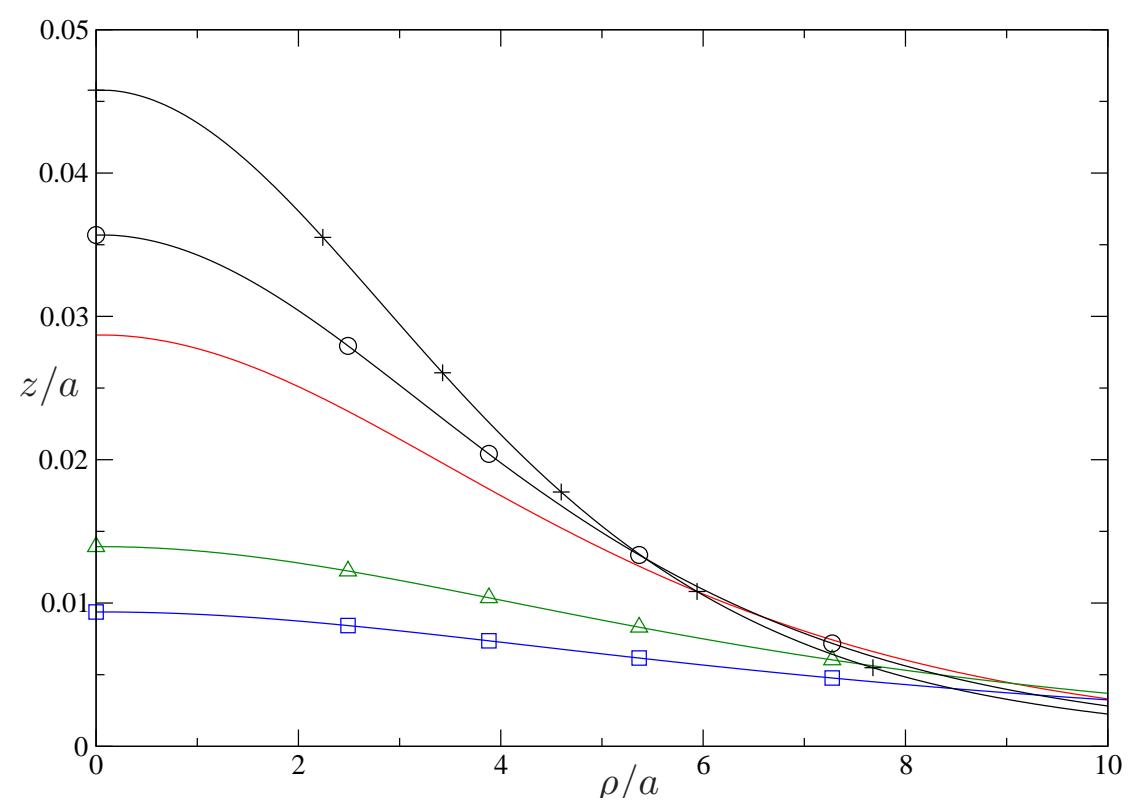

FIG. 4: Free surface shapes at different Bond numbers Bo for a bubble location $l=6 a$. $\mathrm{Bo}=1 / 3(+)$; Bo $=0.1(\circ) ; \mathrm{Bo}=0.05(-)$; Bo $=0.01(\triangle)$; Bo $=0.005(\square)$.
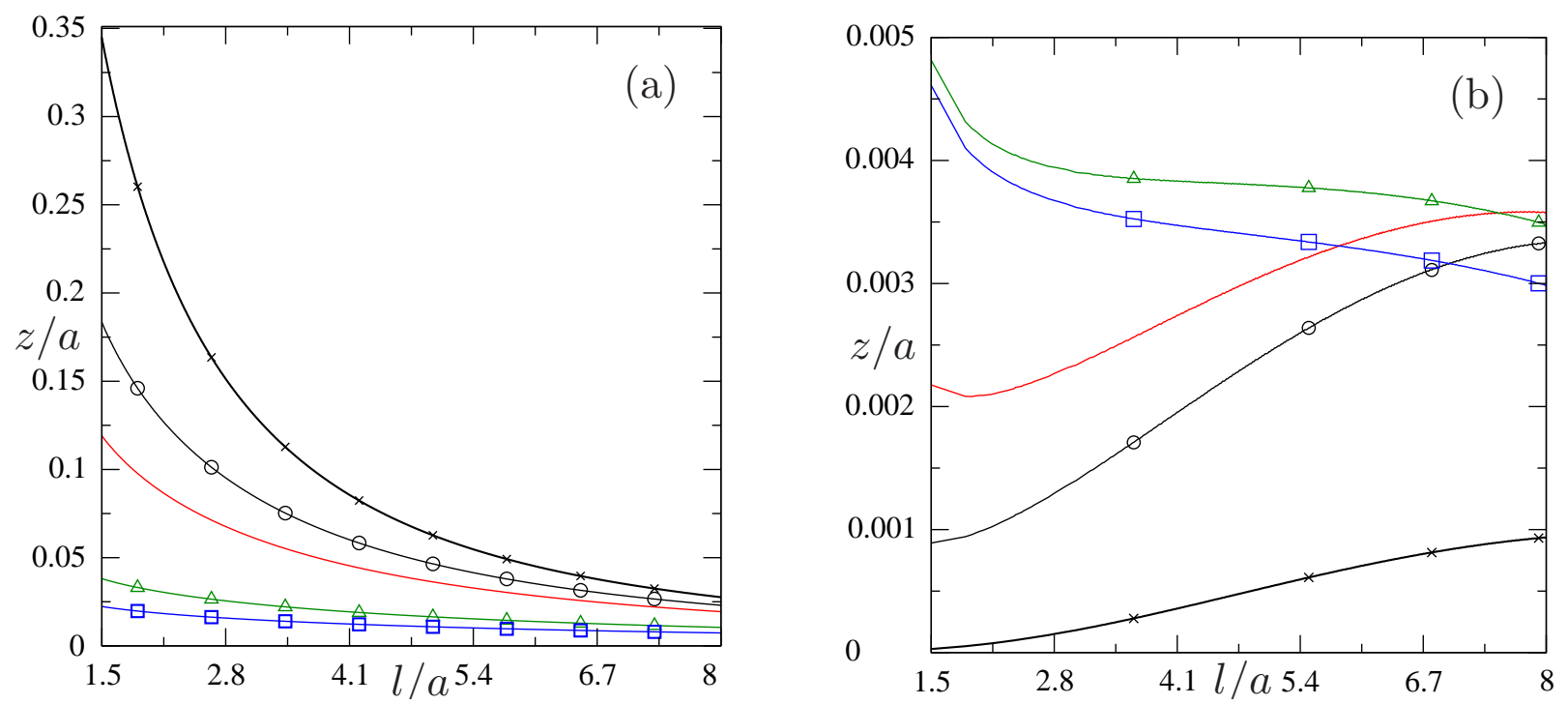

FIG. 5: Free surface deformation $z / a$ versus $l / a$ on the $\rho=0$ axis (a) and at $\rho=10 a(\mathrm{~b})$ for $\mathrm{Bo}=1 / 3(+)$; $\mathrm{Bo}=0.1(\circ)$; $\mathrm{Bo}=0.05(-)$; $\mathrm{Bo}=0.01(\triangle)$; $\mathrm{Bo}=0.005(\square)$. 

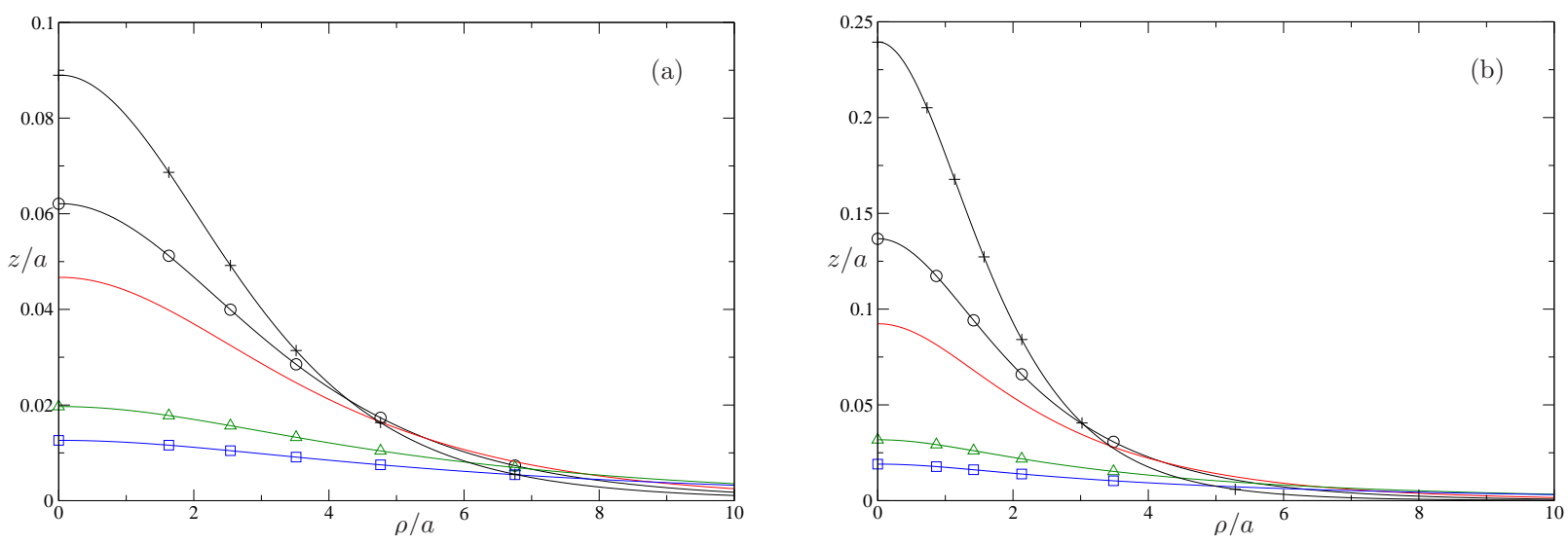

FIG. 6: Free surface shapes at different Bond numbers: Bo $=1 / 3(+)$; Bo $=0.1(\circ)$; $\mathrm{Bo}=0.05(-) ; \mathrm{Bo}=0.01(\triangle) ; \mathrm{Bo}=0.005(\square)$. (a) $l=4 a$ and (b) $l=2 a$.
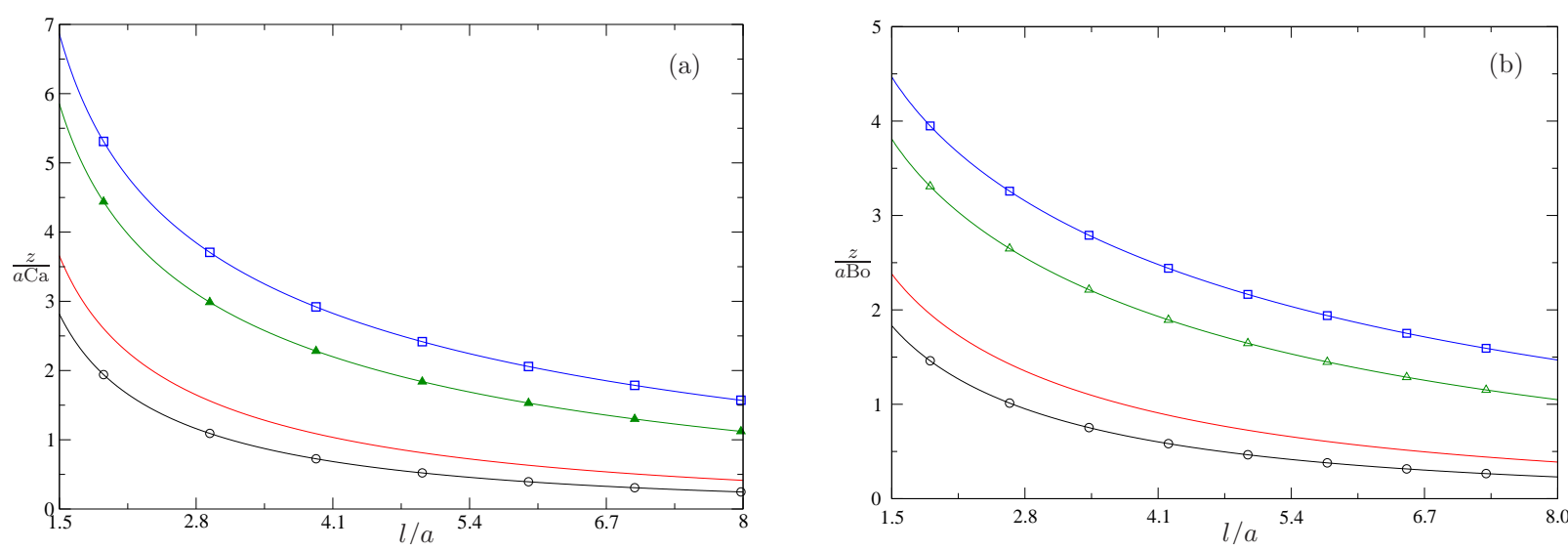

FIG. 7: (a) ratio $z /(a \mathrm{Ca})=2 f(\rho / a)$ and (b) ratio $z /(a \mathrm{Bo})=2 f(\rho / a) / \lambda$ on the $\rho=0$ axis for $\mathrm{Bo}=0.1(\circ) ; \mathrm{Bo}=0.05(-) ; \mathrm{Bo}=0.01(\triangle)$ and $\mathrm{Bo}=0.005(\square)$.

\section{FIRST-ORDER BUBBLE SHAPE}

\section{A. Governing problem for the bubble shape}

Mimicking section $\S$ III A we need to asymptotically enforce at small $\mathrm{Ca}=\mathcal{O}(\mathrm{Bo})$ the relation (4) on the perturbed bubble surface $S_{1}$. Employing bipolar coordinates (recall (15)), as done by Chervenivanova and Zapryanov ${ }^{13}$ for a droplet, is not convenient. As depicted in FIG. 2 and according to Hetsroni and Haber ${ }^{11}$, we instead use spherical coordinates $\left(r^{\prime}, \theta, \phi\right)$, centered at the bubble center-of-volume $O^{\prime}$ such that $\mathbf{O O}^{\prime}=-l \boldsymbol{e}_{z}$, with $\phi \in[0,2 \pi], \theta \in[0, \pi]$ and $r^{\prime}=\left|\boldsymbol{x}^{\prime}\right|$ for $\boldsymbol{x}^{\prime}=\mathbf{O}^{\prime} \mathbf{M}$. For small $\mathrm{Ca}=\mathcal{O}(\mathrm{Bo})$ the nearly-spherical bubble uniform 
pressure $p_{b}$ and axisymmetric surface $S_{1}^{\prime}$ then admit the expansion and equation

$$
p_{b} \sim p_{b}^{0}+\mathrm{Ca} p_{b}^{1}, r^{\prime} \sim a[1+\mathrm{Ca} \xi(\theta)] \text { on } S_{1}^{\prime}
$$

with $p_{b}^{0}=p_{a}+\rho_{l} g l+2 \gamma / a$ prevailing at $\mathrm{Bo}=0$ and unknown uniform pressure $p_{b}^{1}$ and bubble

shape function $\xi(\theta)=\mathcal{O}(1)$. Requiring conservation of the bubble volume and center-ofvolume $O^{\prime}$ location and the bubble surface to be smooth, bounded and to exhibit horizontal tangent planes at its two $\theta=0$ and $\theta=\pi$ poles $^{25}$ provides, at order $\mathcal{O}(\mathrm{Ca})$, the conditions

$$
\begin{aligned}
& \int_{0}^{\pi} \xi(\theta) \sin \theta d \theta=0, \quad \int_{0}^{\pi} \xi(\theta) \sin \theta \cos \theta d \theta=0 \\
& \quad \xi \text { and } \frac{d \xi}{d \theta} \text { bounded in }[0, \pi] ; \frac{d \xi}{d \theta}=0 \text { for } \theta=0, \pi .
\end{aligned}
$$

As for the free surface, we set $\overline{\boldsymbol{\nabla}}_{S} \cdot \boldsymbol{n}=d \boldsymbol{\nabla}_{S} \cdot \boldsymbol{n}$ and $\overline{\boldsymbol{\sigma}}=d \boldsymbol{\sigma} /(\mu U)$ when imposing (4) while this time the second approximation $(26)$ yields $\left(\operatorname{see}^{11},{ }^{26}\right)$

$$
\overline{\boldsymbol{\nabla}}_{S} \cdot \boldsymbol{n} \sim 4-2 \mathrm{Ca} L(\xi), L(\xi)=2 \xi+\frac{1}{\sin \theta} \frac{d}{d \theta}\left(\sin \theta \frac{d \xi}{d \theta}\right)
$$

As shown in Appendix B, enforcing (4) up to order $\mathcal{O}(1)$ then gives the pressure $p_{b}^{1}$ (see Appendix B) and for the bubble shape function $\xi$ the linear second-order differential equation

$$
\begin{aligned}
& t_{b} \xi \sin \theta \cos \theta+2 \xi \sin \theta+\frac{d}{d \theta}\left[\sin \theta \frac{d \xi}{d \theta}\right]=R(\theta) \sin \theta, t_{b}=3 \text { Bo, } \\
& R(\theta)=\frac{1}{2}\left\{\frac{1}{2} \int_{0}^{\pi}\left[\boldsymbol{n}_{0} \cdot \overline{\boldsymbol{\sigma}}_{0} \cdot \boldsymbol{n}_{0}\right](\alpha) \sin \alpha d \alpha-6 \lambda_{0} \cos \theta-\left[\boldsymbol{n}_{0} \cdot \overline{\boldsymbol{\sigma}}_{0} \cdot \boldsymbol{n}_{0}\right](\theta)\right\} .
\end{aligned}
$$

In (31) we calculate $\lambda_{0}$ from (A4) and the occurring normalized zeroth-order normal traction is given by $\left[\boldsymbol{n}_{0} \cdot \overline{\boldsymbol{\sigma}}_{0} \cdot \boldsymbol{n}_{0}\right](\theta)=d\left[\sigma_{0 \zeta \zeta}\left(-\zeta_{p}, \eta\right)\right] /(\mu U)$ with, see $(\mathrm{A} 5), \cos \eta=\left(\cos \theta \cosh \zeta_{p}-\right.$ 1) $/\left(\cos \theta-\cosh \zeta_{p}\right)$. Accordingly, $R$ and $\xi$ solely depend on $l / a$ and $(l / a$, Bo), respectively. In summary, $\xi$ is obtained by solving (30) and (27)-(28) for $R$ given by (31).

\section{B. Approximated well-posed and ill-posed problems}

Setting $x=\cos \theta$, we seek the bubble shape function $f(x)=\xi(\theta)$ for $x$ in $[-1,1]$. Using the prime symbol for differentiation with respect to $x$ and the relations

$$
f(x)=\xi(\theta), \frac{d \xi}{d \theta}=-\sqrt{1-x^{2}} f^{\prime}(x)
$$


makes it possible to recast the determination of $\xi$ into the following equivalent problem

$$
\begin{aligned}
& \left(1-x^{2}\right) f^{\prime \prime}-2 x f^{\prime}+\left(2+t_{b} x\right) f=R(x) \text { for }-1<x<1, \\
& \int_{-1}^{1} f(x) d x=0, \int_{-1}^{1} x f(x) d x=0, \\
& f \text { and } \sqrt{1-x^{2}} f^{\prime}(x) \text { are bounded in }[-1,1] \\
& \lim _{x \rightarrow-1} \sqrt{1-x^{2}} f^{\prime}(x)=0, \lim _{x \rightarrow 1} \sqrt{1-x^{2}} f^{\prime}(x)=0 .
\end{aligned}
$$

As will be shown in $\S$ IV B 2, such a problem turns out to be ill-posed for $t_{b}>0$.

\section{Approximated well-posed problem for $t_{b}=0$}

For $t_{b}=0$ it is possible to solve (33)-(36) by expanding $f$ in Legendre polynomials $P_{n}^{23}$, as done by Hetsroni and Haber ${ }^{11}$ when dealing with the weakly deformation of a bubble freelysuspended in a prescribed arbitrary ambiant Stokes flow in an unbounded liquid. Exploiting the differential equation satisfied by each Legendre polynomial and the properties ${ }^{23}$

$$
P_{0}(x)=1, P_{1}(x)=x, \int_{-1}^{1} P_{n}(x) P_{m}(x) d x=\frac{2 \delta_{n m}}{2 n+1}
$$

with $\delta_{n m}$ the usual Kronecker delta, we easily arrive at the desired solution

$$
f(x)=\sum_{n \geq 2} L_{n} P_{n}(x), \quad L_{n}=\frac{2 n+1}{2\left[2-n-n^{2}\right]} \int_{-1}^{1} P_{n}(x) R(x)(x) d x \text { for } n \geq 2 .
$$

We also numerically solved (33)-(36) using a $\mathcal{O}\left(h^{2}\right)$ second-order centered Finite-Difference Method (FDM) with $N-1$ nodal points $x_{n}=-1+n h$ in $]-1,1[$ (with $n=1, \cdots, N-1, h=$ $2 / N)$ and discretizing the integral conditions (34) also at $\mathcal{O}\left(h^{2}\right)$ using a trapezoidal rule. As

illustrated in TABLE II, comparisons of the implemented FDM against (38) are excellent and the FDM exhibits the expected $\mathcal{O}\left(h^{2}\right)$ accuracy.

\section{Ill-posed problem for $t_{b}>0$}

For $t_{b}>0$ there is no guarantee that our requirements (35)-(36) are consistent with (33)-(34). This issue is addressed by first numerically solving (33)-(34) and then checking whether (35)-(36) are satisfied. To do so we again run a $\mathcal{O}\left(h^{2}\right)$ Finite-Difference Method solely differing by a minor change (one had just to code the extra term $t_{b} x f$ arising in (33)) from the one validated for the previous $t_{b}=0$ case. 


\begin{tabular}{cccc}
\hline$x$ & $\mathrm{FDM}, h=0.01$ & $\mathrm{FDM}, h=0.001$ & Legendre \\
\hline-0.95 & -0.0515542907 & -0.0515508201 & -0.0515507859 \\
-0.30 & 0.0211203840 & 0.0211179167 & 0.0211178918 \\
0. & 0.0328849811 & 0.0328815520 & 0.0328815173 \\
0.30 & 0.0273688098 & 0.0273649720 & 0.0273649332 \\
0.95 & -0.0636789022 & -0.0636769410 & -0.0636769211 \\
\hline
\end{tabular}

TABLE II: Computed function $f(x)$ for $t_{b}=0$ and $l=3 a$ when retaining $N_{t}=25$ terms in expansions (A1) and (A4). In the Legendre approach (38) we take $2 \leq n \leq 20$ and evaluate each coefficient $L_{n}$ with a Gaussian integration scheme at a $10^{-8}$ accuracy level.
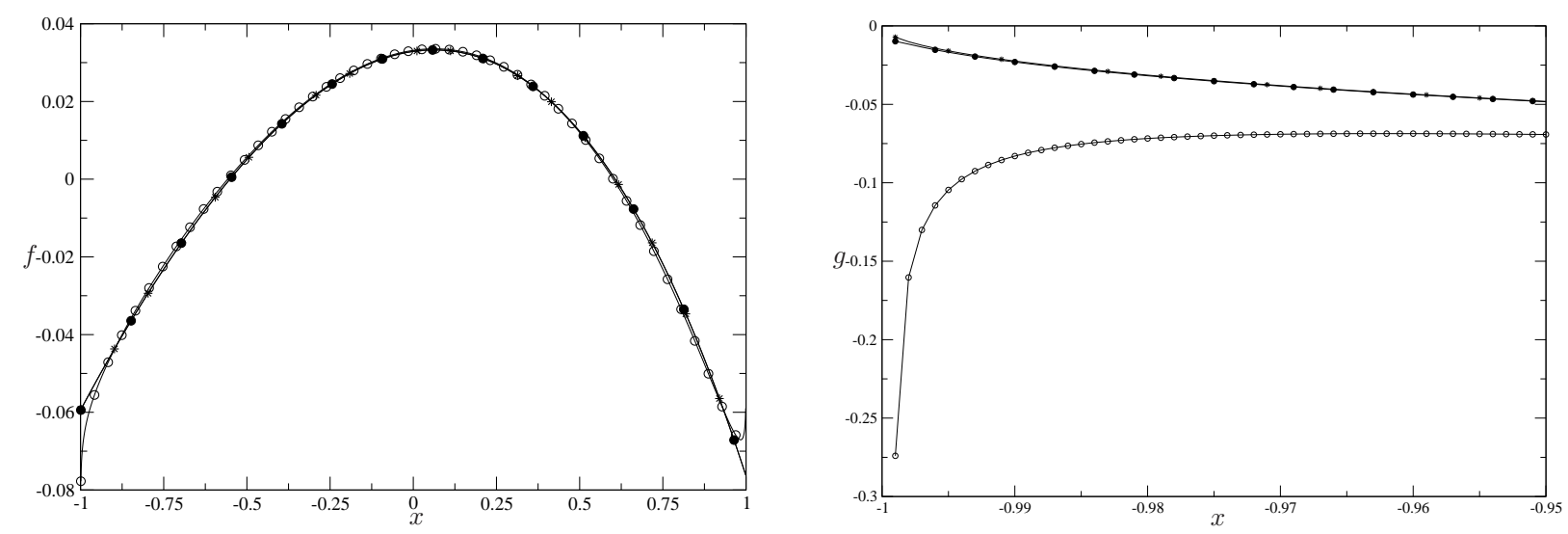

FIG. 8: Numerical solution to (33)-(34) for the bubble location $l=3 a$, spacing $h=0.001$ and $t_{b}=1(\circ), t_{b}=0.01(\bullet)$ or $t_{b}=0(*)$. (a) Shape function $\xi=f$. (b) Function $g(x)=-\sqrt{1-x^{2}} f^{\prime}(x)$ for $x$ in the range $[-0.999,-0.95]$.

Both computed functions $f$ and $g(x)=-\sqrt{1-x^{2}} f^{\prime}(x)$ are plotted versus $x$ in FIG. 8 for $h=10^{-3}$, a bubble location $l=3 a$ (still keeping $N_{t}=25$ terms in expansions (A1) and (A4)) and different values of $t_{b} \geq 0$. As seen in FIG. 8(a), $f$ is not only bounded (i.e. the first requirement (35) holds) but it is also nearly-insensitive to the value of the parameter $t_{b}$ except near the $x=-1,1$ end points where $f$ also exhibits a large derivative. Moreover, as illustrated near the $x=-1$ point (i.e. close to the south pole) in FIG. 8(b), the function $g$ is bounded in $[-1,1]$. Therefore, the computed solution $f$ satisfies (33)-(35) whatever $t_{b} \geq 0$. However, (36) is not fulfilled because $g$ is non-zero at $x=-1,1$ for $t_{b}>0$ although it tends to zero there as $t_{b}$ vanishes (see FIG. 8(b) for $x \rightarrow-1$ if $t_{b}=0.01$ ). 

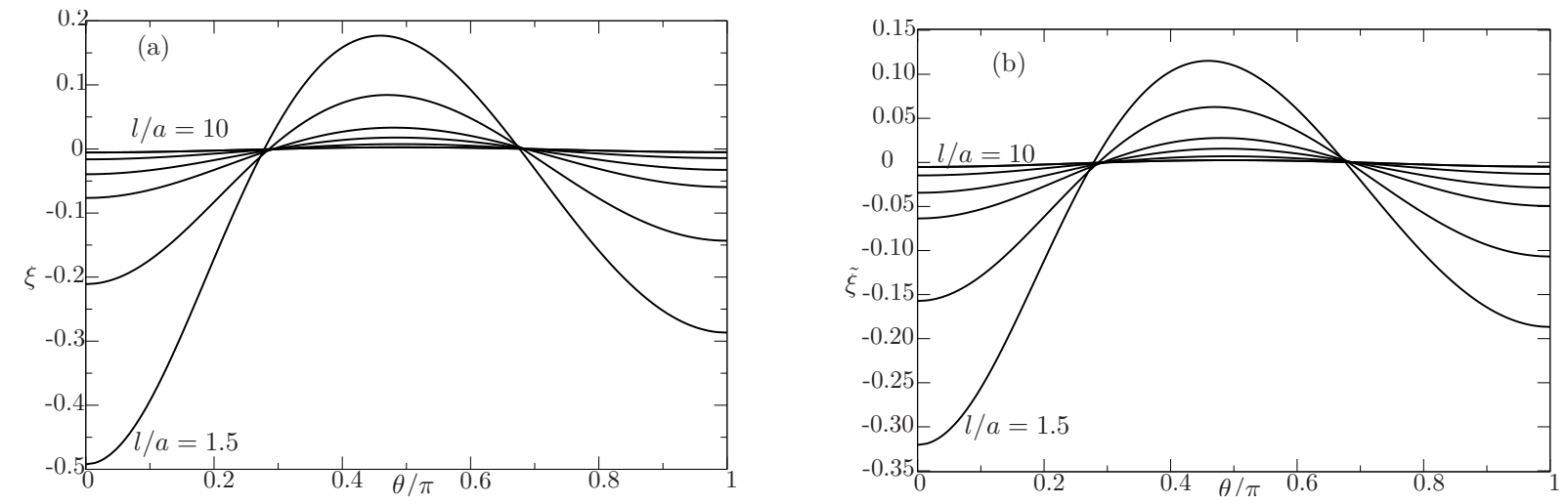

FIG. 9: Functions $\xi$ and $\tilde{\xi}=\xi / \lambda$ versus $\theta / \pi$ for $l / a=1.5,2,3,4,6$ and 10 (taken negative values at $\theta=0$ decrease with $l / a$ ). (a) function $\xi$. (b) function $\tilde{\xi}$.

In summary, our computations reveal that (33)-(36) is ill-posed for $t_{b}>0$. However, for small $t_{b}>0$ the solution $f$ to (33)-(36) is close to the regular one obtained for $t_{b}=0$ and we thus henceforth compute the perturbed bubble shape by taking $t_{b}=0$.

\section{Numerical results and discussion}

In this section perturbed bubble shapes are computed with spacing $h=10^{-3}$ and $t_{b}=0$. Since in practice we give Bo it is worth introducing the function $\tilde{\xi}$ such that, recalling (26), Bo $\tilde{\xi}=\mathrm{Ca} \xi$. Because $\mathrm{Bo}=\lambda_{0}$ Ca note that $\tilde{\xi}=\xi / \lambda_{0}$. As already pointed out, taking $t_{b}=0$ make $\xi$ and therefore also $\tilde{\xi}$ solely depend upon the bubble location $l / a$. Both functions are plotted in FIG. 9 versus $\theta / \pi$ for $l / a=1.5,2,3,4,6$ and 10. Not surprisingly, the bubble is squeezed by its weak interaction with the free surface near its $\theta=0$ north pole and forced to expand away from its axis of revolution for $\theta$ close to $\pi / 2$. As a result, $\xi$ and $\tilde{\xi}$ are negative and positive near $\theta=0$ or near $\theta=\pi / 2$, respectively. Moreover, the bubble is also squeezed near its $\theta=\pi$ south pole (negative functions near this pole) the deformation being there smaller than near the north pole because the interacting free surface is more distant (one bubble diameter more). Finally, domains of positive and negative values of the shape functions $\xi$ and $\tilde{\xi}$ are nearly insensitive to the bubble location $l / a$.

The bubble deformation is weak when compared with the free surface deformation on the $\left(z^{\prime} O z\right)$ axis. This is clear when comparing for a prescribed bubble location $l / a$ the quantities $z /(a \mathrm{Ca})$ at $\rho=0$ (recall FIG. $7(\mathrm{a}))$ and $\xi$ at the $\theta=0$ north pole. Moreover, $|\tilde{\xi}|$ is at the most of order $7 \%$ as soon as $l$ exceeds $3 a$. Therefore, the perturbed bubble shapes remain 


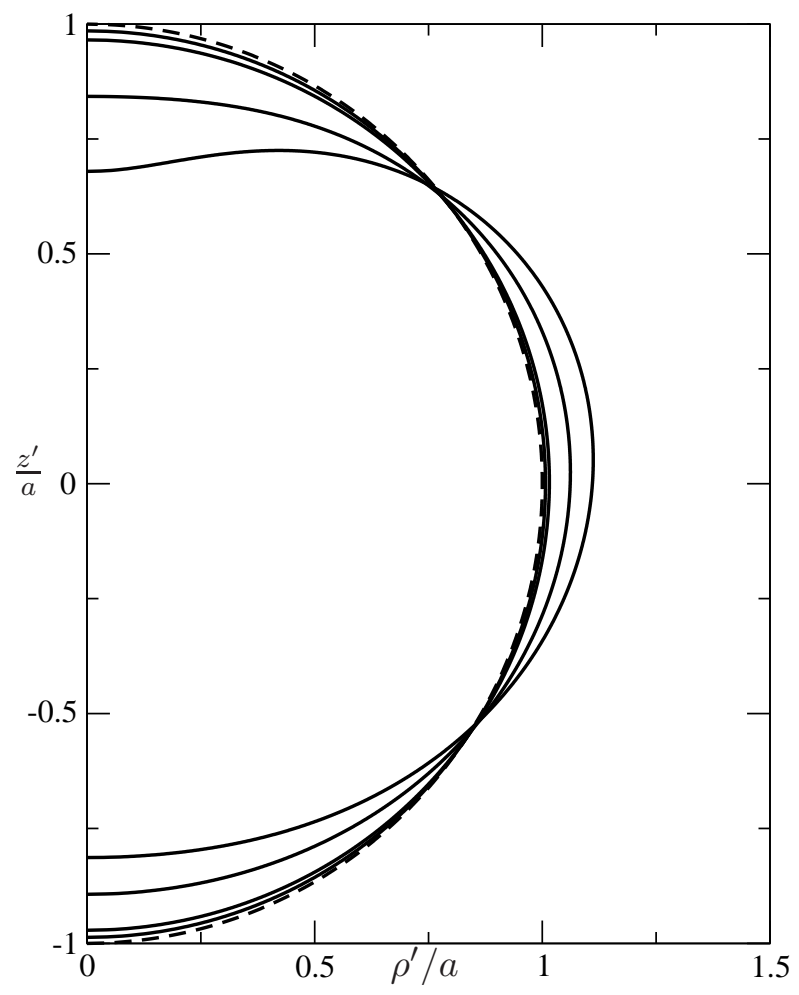

FIG. 10: Normalized half spherical bubble (dashed line) and several exaggerated perturbed bubble profiles (solid lines) for $r^{\prime} / a=1+\tilde{\xi}(\theta)$ and $l / a=1.5,2,4,6$. For each profile the value of $l / a$ is obtained knowing that at $z^{\prime}=0$ the bubble deformation increases with $a / l$.

very close to a sphere with radius $a$ as soon as Bo $\leq 0.2$ even for $l / a=1.5$. This is why in FIG. 10 we deliberately show exaggerated half (due to the symmetry about the $\left(z^{\prime} O z\right)$ axis) deformed bubble profiles by setting Bo $=1$ and thus taking $r^{\prime} / a=1+\tilde{\xi}(\theta)$. Those profiles are drawn for $l / a=1.5,2,4,6$ in the half normalized plane $\left(\rho^{\prime} / a, z^{\prime} / a\right)$ where $z^{\prime}=z+l$ (the bubble center of volume $O^{\prime}$ having in this plane coordinates $(0,0)$ whatever $l / a$ ). Such "amplified" profiles show a bubble squeezed at its north and south poles and expanded in the vicinity of its horizontal $z^{\prime}=0$ plane.

\section{COMPARISONS AGAINST AN AXISYMMETRIC BEM SOLUTION}

As mentioned in the introduction, direct numerical computations have been recently performed ${ }^{10,27}$ to track in time the free surface and bubble boundary locations in a large range of Bond number Bo. Those direct simulations appeal to an axisymmetric Boundary Element Method (BEM) solution which has been actually found, as reported in Pigeonneau 


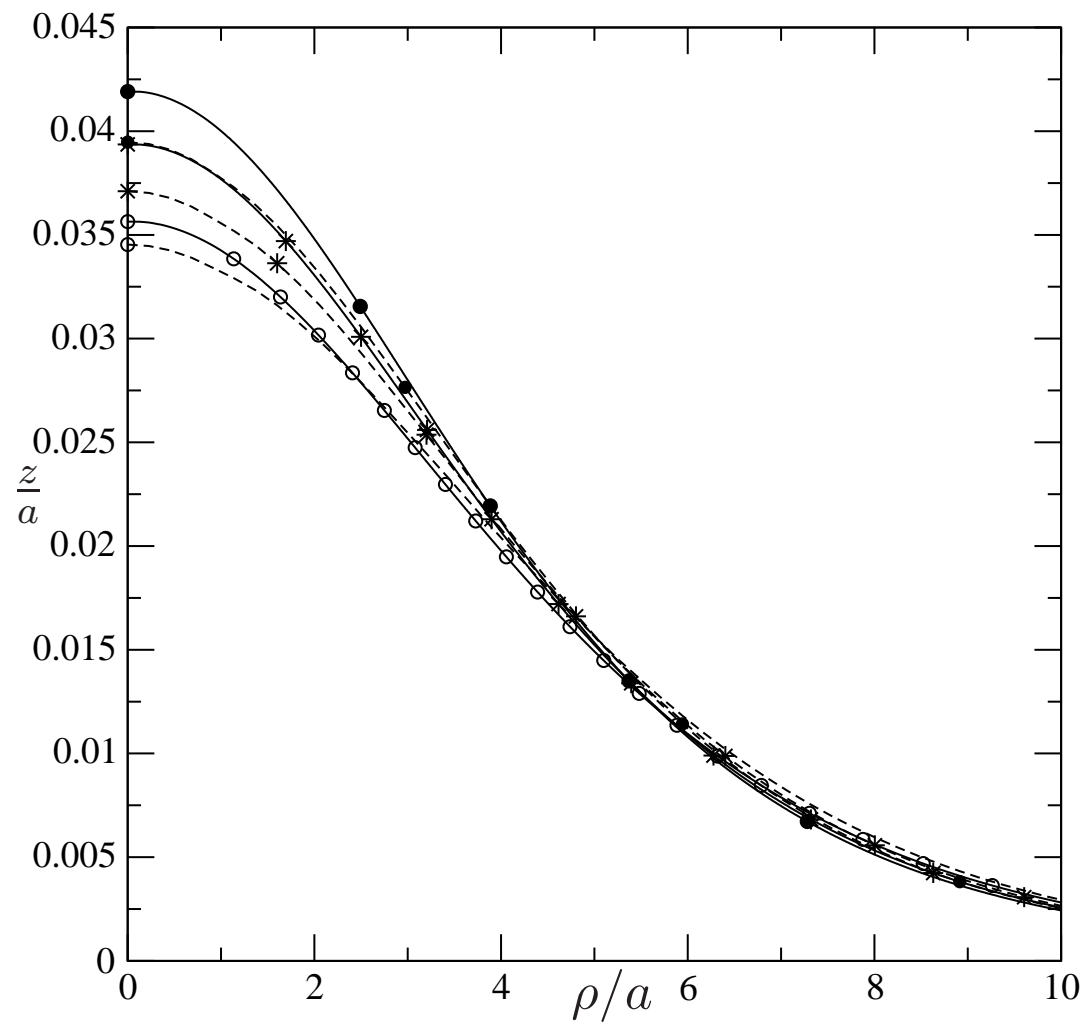

FIG. 11: Free surface shapes from the BEM solution (dashed lines) and the first-order asymptotic analysis (solid lines) at $\mathrm{Bo}=0.1(\mathrm{\circ})$ and $\mathrm{Bo}=0.2(\bullet)$. The bubble center of volume is located at $l / a \sim 6$ (see TABLE III for the associated 4-digit value of $l /(2 a)$ ).

and Sellier ${ }^{10}$, to experience numerical accuracy troubles for Bo $\leq \mathcal{O}(0.1)$. This section compares at small Bond number Bo the weakly perturbed free surface and bubble surface shapes predicted either by the BEM or the present asymptotic analysis.

\section{A. Comparisons for the weakly perturbed free surface}

Predictions of the asymptotic analysis developed in $\S$ III and the Boundary Element Method (BEM) are compared by running the asymptotic procedure for the bubble centerof-volume location $l / a$ computed by the BEM solution. First we draw in FIG. 11 the resulting free surface shapes $z / a$ versus $\rho / a$ for a bubble location $l / a \sim 6$ and $\mathrm{Bo}=0.1,0.2$. The asymptotic and BEM results are compared by inspecting the quantity

$$
\Delta=\max \left|(z / a)_{\mathrm{BEM}}-(z / a)_{\text {Asymptotic }}\right|
$$




\begin{tabular}{ccccccc}
\hline$(l /(2 a), \mathrm{Bo})$ & $(3.0008,0.1)$ & $(3.0003,0.2)$ & $(2.0022,0.1)$ & $(2.0022,0.2)$ & $(1.0016,0.1)$ & $(1.0021,0.2)$ \\
\hline$(\mathrm{Ca})^{2}$ & $8.40 \cdot 10^{-3}$ & $3.36 \cdot 10^{-2}$ & $7.70 \cdot 10^{-3}$ & $3.06 \cdot 10^{-2}$ & $5.60 \cdot 10^{-3}$ & $2.22 \cdot 10^{-2}$ \\
$\Delta$ & $1.10 \cdot 10^{-3}$ & $2.40 \cdot 10^{-3}$ & $3.30 \cdot 10^{-3}$ & $8.00 \cdot 10^{-3}$ & $2.73 \cdot 10^{-2}$ & $2.14 \cdot 10^{-2}$ \\
$(\rho / a)_{m}$ & 0 & 0 & 4 & 0 & 2.3 & 0 \\
\hline
\end{tabular}

TABLE III: Setting $(l /(2 a), \mathrm{Bo})$ and quantities $(\mathrm{Ca})^{2}, \Delta$ and $(\rho / a)_{m}$ for FIG. 11-12.
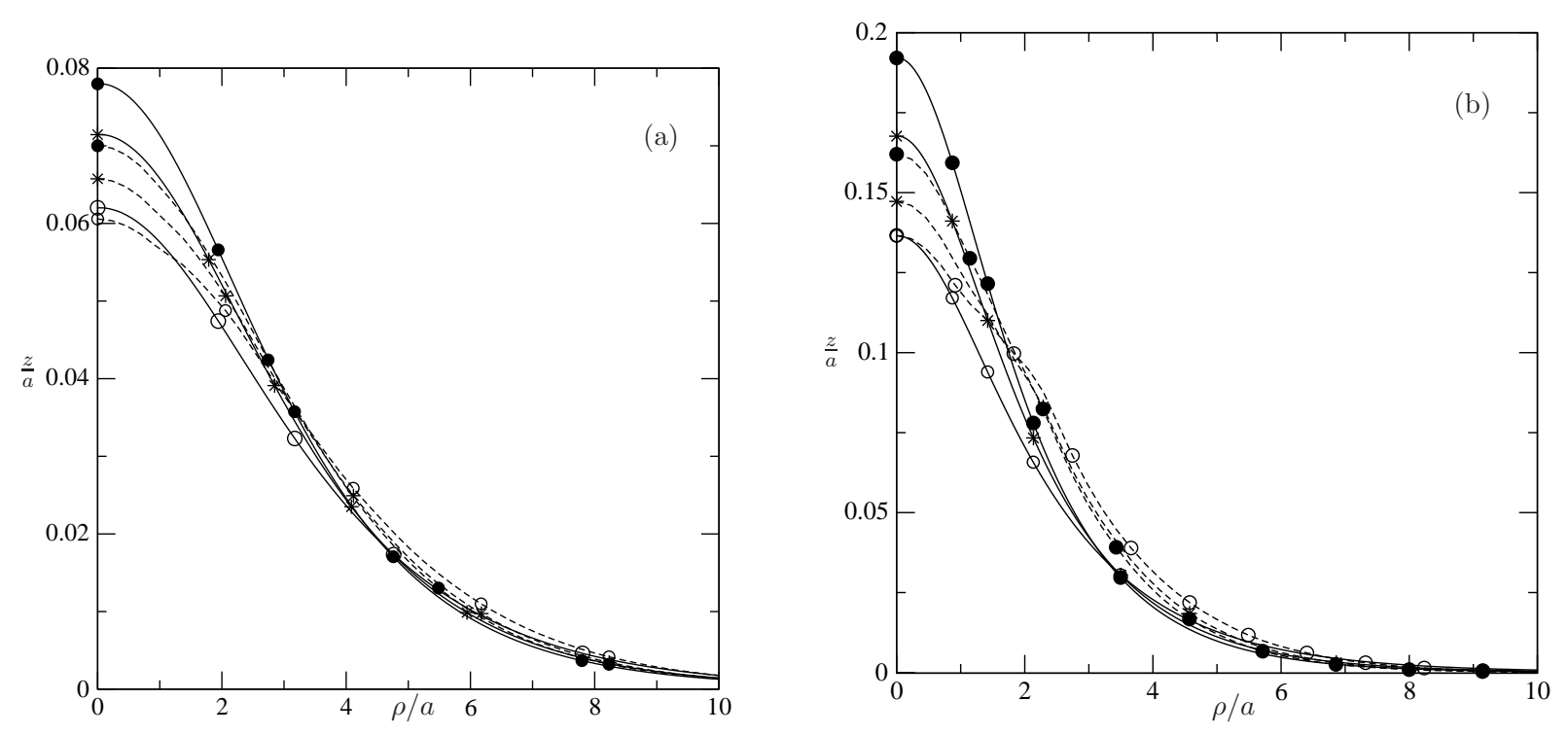

FIG. 12: Free surface shapes from the BEM solution (dashed lines) and the first-order asymptotic analysis (solid lines) at $\mathrm{Bo}=0.1(\circ), \mathrm{Bo}_{0}=0.15\left(^{*}\right)$ and $\mathrm{Bo}=0.2(\bullet)$. Here $l / a \sim 4$ (a) or $l / a \sim 2$ (b) (see also TABLE III for the associated 4-digit values of $l /(2 a)$ ).

versus the small quantity $(\mathrm{Ca})^{2}$. Note that $\Delta$ is actually reached at a not-necessarily zero location $(\rho / a)_{m}$. The values of $(\rho / a)_{m}, \Delta$ and $(\mathrm{Ca})^{2}$ for FIG. 11 (and also for FIG. 12) are given in TABLE III which also provides the 4-digit value of the bubble center-of-volume location $l /(2 a)$ obtained by the BEM computations and used in the asymptotic analysis. At $l \sim 6 a$ a nice agreement between the asymptotic and BEM is found for Bo in the range $[0.1,0.2]$. Of course, at a given Bond number the free surface is more perturbed when the bubble approaches and this suggests also checking our asymptotic results for $l<6 a$. This has been done for $l / a \sim 4,2$ in FIG. 12 and is also quantified in TABLE III.

While the $l \sim 4 a$ results are still in full agreement some discrepancies are found at Bo $=0.1$ for the $l \sim 2 a$ case of a close bubble. For this pair (Bo, $l / a)$ the computed BEM free surface exhibits a non-physical weavy shape close the $\left(z^{\prime} O z\right)$ axis (inspect FIG. 12(b)) 

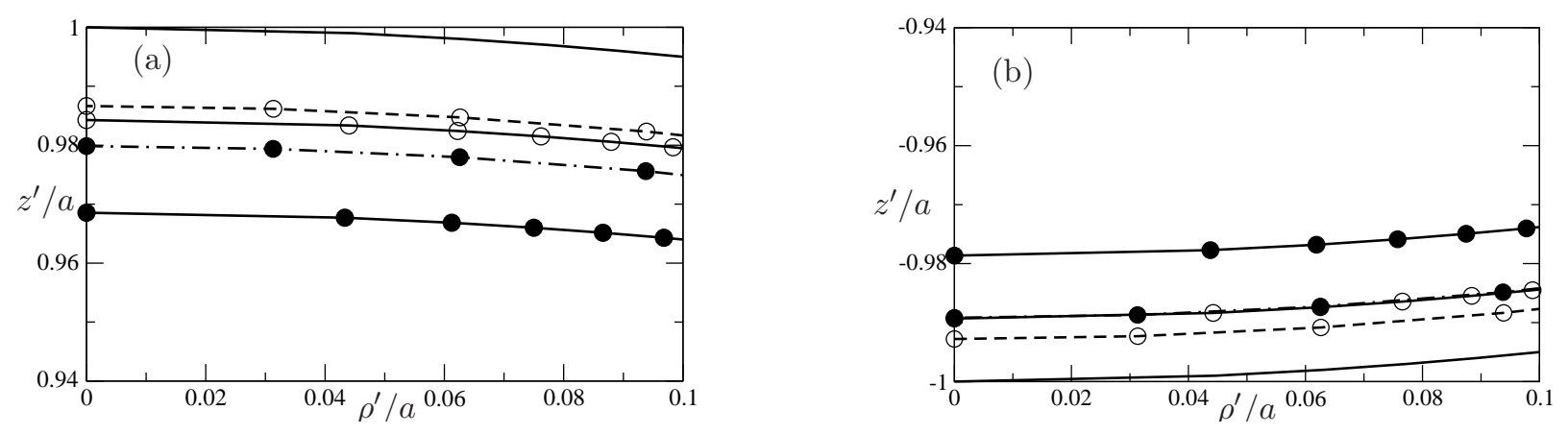

FIG. 13: Parts of half bubble normalized profiles from the BEM Code (dashed lines) and first-order asymptotic analysis (solid lines) at $\mathrm{Bo}=0.1$ (०) and $\mathrm{Bo}=0.2(\bullet)$. Here $l / a \sim 2$ (see TABLE III) and the normalized unperturbed spherical bubble profile is shown in solid line. (a) Bubble north pole $\left(z^{\prime}=a\right)$ vicinity. (b) Bubble south pole $\left(z^{\prime}=-a\right)$ vicinity.

and this results in a value of $\Delta$ (see TABLE III) which is much larger than $(\mathrm{Ca})^{2}$. This case illustrates the troubles experienced at low Bond number by the BEM computations for a close bubble, i.e. when on each deformed surface the local curvature slightly differs from the uniform one prevailing for unperturbed surfaces (see also the introduction).

\section{B. Case of the bubble shape}

Comparisons between the BEM and asymptotic approaches have been also made and found to be very convincing for the bubble shape. As noted in $\S$ IV C, the bubble deformation is small when compared to the free surface shape deformation. Accordingly and in contrast to the case depicted in FIG. 12(b) for the perturbed free surface, the agreement between the BEM and asymptotic bubble shapes remains quite good even at $l / a=2$ and $\mathrm{Bo}=0.2$. This is illustrated by drawing in FIG. 13 and in FIG. 14 the perturbed bubble normalized half profile where the largest deformations arise, i.e. near its north and south poles and equatorial plane.

As seen in those figures, the asymptotic analysis yields a larger deformation than the BEM approach. Moreover, the difference (when normalized with $a$ ) between those methods on the entire bubble profile is order of $4 \cdot 10^{-3}$ or $12 \cdot 10^{-3}$ for Bo $=0.1$ or Bo $=0.2$, respectively. Recalling the values given in TABLE III for the associated values of $\mathrm{Ca}^{2}$ then shows that, as announced, both predictions well agree to order $\mathcal{O}\left(\mathrm{Ca}^{2}\right)$. 


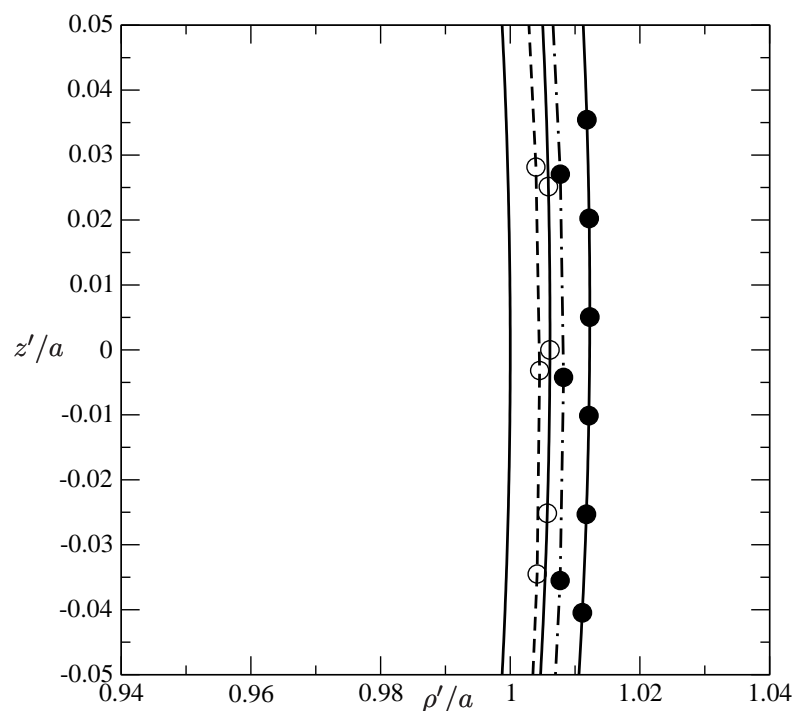

FIG. 14: Vicinity of the $z^{\prime}=0$ half equatorial plane for the normalized deformed bubble profiles from the BEM Code (dashed lines) and first-order asymptotic analysis (solid lines) at $\mathrm{Bo}=0.1(\circ)$ and $\mathrm{Bo}=0.2(\bullet)$. The bubble center of volume is located as in FIG. 13 (with $l / a \sim 2$ ) and the normalized unperturbed spherical bubble profile is shown in solid.

\section{CONCLUSIONS}

The shapes of interacting free surface and bubble in presence of a uniform gravity field have been asymptotically obtained at the first order in small Bond number Bo (or equivalently capillary number $\mathrm{Ca}$ ). The analysis appeals to the accurate determination of the normal stress $\boldsymbol{n}_{0} \cdot \boldsymbol{\sigma}_{0} \cdot \boldsymbol{n}_{0}$ prevailing in the case of unperturbed bubble spherical surface and flat free surface. The gap of each surface to its unperturbed location is measured by the quantity $\mathrm{Ca} f$ with $f=\mathcal{O}(1)$ the associated shape function obeying a linear problem involving an ordinary differential equation in which the gravity appears through a term proportional to Bo $f$. Keeping this latter term yields either a well-posed or an ill-posed problem (in contrast to the unbounded liquid Haber ${ }^{11}$ ) for the free surface or bubble, respectively. The bubble problem however is well posed if one ignores the requirement of horizontal tangent plane at the bubble north and south poles or discards the Bo $f$ term both choices predicting for small Bond number Bo close bubble shape functions except in the very vicinity of the bubble north and south poles. In the present work the Bo $f$ term is ignored in the bubble shape problem. The asymptotic analysis numerical implementation reveals that, at given bubble location and small Bond number, the free surface is in practice 
more perturbed than the bubble. It also well agree with the BEM computations ${ }^{10}$ in the overlapping range $\mathrm{Bo}=\mathcal{O}(0.1)$.

One can readily deal with interacting bubble and free surface having unequal uniform surfaces tensions by taking in the present analysis for each surface Bond and Capillary numbers (recall (8)) based on the addressed boundary surface tension. Finally, one may think about extending the work to the case of several bubbles interacting, at small Bond number and in axisymmetric configuration, with a free surface. This time the evaluation of the zeroth-order normal stress $\boldsymbol{n}_{0} \cdot \boldsymbol{\sigma}_{0} \cdot \boldsymbol{n}_{0}$ on the unperturbed flat free surface and on each spherical bubble boundary might be done by proposing a new BEM approach somewhat combining the ones employed in Sellier ${ }^{28}$ for the gravity-driven motion of a cluster of spherical bubbles in an unbounded liquid and in Pasol et al. ${ }^{29}$ for a solid sphere interacting with a fluid-fluid interface. Since this challenging task requires substantial additional efforts, it is postponed to a future work.

\section{Appendix A: Zeroth-order flow and drag coefficient}

Denoting by $P_{n}$ the usual Legendre polynomial of order $n^{30}$, the stream function $\psi$ reads ${ }^{31}$

$$
\begin{aligned}
& \psi(\zeta, \chi)=U(\cosh \zeta-\chi)^{-\frac{3}{2}} \sum_{n=1}^{\infty} U_{n}(\zeta)\left[P_{n-1}(\chi)-P_{n+1}(\chi)\right], \\
& U_{n}(\zeta)=B_{n} \sinh [(n-1 / 2) \zeta]+D_{n} \sinh [(n+3 / 2)] \\
& B_{n}=\frac{(2 n+3) k_{n}\left[e^{2 \zeta_{p}}-e^{-(2 n+1) \zeta_{p}}\right]}{\cosh (2 n+1) \zeta_{p}-\cosh 2 \zeta_{p}}, D_{n}=\frac{(2 n-1) k_{n}\left[e^{-(2 n+1) \zeta_{p}}-e^{-2 \zeta_{p}}\right]}{\cosh (2 n+1) \zeta_{p}-\cosh 2 \zeta_{p}}
\end{aligned}
$$

with $k_{n}=c^{2} n(n+1) /[\sqrt{2}(2 n+1)(2 n+3)(2 n-1)]$ for $n \geq 1$. The drag coefficient $\lambda_{0}$, defined by (13), receives the analytical form ${ }^{17} \lambda_{0, a}$ given by

$$
\lambda_{0, a}=\left[\sqrt{2} \sum_{n=1}^{\infty}(2 n+1)\left(B_{n}+D_{n}\right)\right] /(2 a c)
$$

but can also be evaluated by integrating the normal traction $\sigma_{0 \zeta \zeta}$ either on $S_{0}^{\prime}(\zeta=0)$ or on $S_{1}^{\prime}\left(\zeta=-\zeta_{p}\right)$. As the reader may easily check, upon introducing

$$
\begin{aligned}
& \lambda_{0,1}=-\frac{a}{2 \mu U} \int_{0}^{\pi} \sigma_{0 \zeta \zeta}\left(-\zeta_{p}, \eta\right) \cos \theta \sin \theta d \theta, \cos \eta=\frac{\cos \theta \cosh \zeta_{p}-1}{\cos \theta-\cosh \zeta_{p}} \\
& \lambda_{0,0}=-\frac{1}{2 \mu U a} \int_{0}^{\infty} \rho \sigma_{0 \zeta \zeta}(0, \eta) d \rho=-\frac{a\left(\sinh \zeta_{p}\right)^{2}}{2 \mu U} \int_{0}^{\pi} \frac{\sigma_{0 \zeta \zeta}(0, \eta) \sin \eta}{(1-\cos \eta)^{2}} d \eta
\end{aligned}
$$


with angle $\theta$ defined in FIG. 2 one indeed arrives at $\lambda_{0}=\lambda_{a, 0}=\lambda_{0,1}=\lambda_{0,0}$. These relations are numerically checked in TABLE I, for several bubble locations $l / a$, by retaining $N_{t}$ terms in (A1) and (A4) and performing each integration in (A5)-(A6) at a $10^{-14}$ accuracy using an iterative scheme with Gaussian quadratures.

\section{Appendix B: Bubble first-order pressure and shape function problem}

Enforcing (4) and using (29) provides on the unperturbed bubble boundary $r=a$

$$
\frac{1}{\mathrm{Ca}}\left[4+\frac{d}{\gamma}\left(p_{a}+\rho_{l} g l-p_{b}^{0}\right)\right] \sim 6 \mathrm{Bo} \xi \cos \theta+2 L(\xi)-2 R(\theta)
$$

with angle $\theta$ introduced in FIG. 2 and, using the link $\mathrm{Bo}=\lambda_{0}$ Ca, the following function

$$
R(\theta)=-\frac{1}{2}\left\{\frac{d}{\gamma} p_{b}^{1}+6 \lambda_{0} \cos \theta+\left[\boldsymbol{n}_{0} \cdot \overline{\boldsymbol{\sigma}}_{0} \cdot \boldsymbol{n}_{0}\right](\theta)\right\} .
$$

in which $\left[\boldsymbol{n}_{0} \cdot \overline{\boldsymbol{\sigma}}_{0} \cdot \boldsymbol{n}_{0}\right](\theta)$ means the normalized zeroth-order normal stress applied on the unperturbed bubble surface $S_{1}^{\prime}$ at point such that $z=-l+a \cos \theta$. Enforcing (B1) at $\mathcal{O}\left(\mathrm{Ca}^{-1}\right)$ and $\mathcal{O}(1)$ retrieves $p_{b}^{0}$ and gives (30), respectively. Now integrating (30) over $\theta$ in $[0, \pi]$ and using (27)-(28) yields the compatibility relation

$$
\int_{0}^{\pi} R(\theta) \sin \theta d \theta=0
$$

Owing to the definition (B2), the condition (B3) provides the first-order pressure

$$
p_{b}^{1}=-\frac{\gamma}{2 d} \int_{0}^{\pi}\left[\boldsymbol{n}_{0} \cdot \overline{\boldsymbol{\sigma}}_{0} \cdot \boldsymbol{n}_{0}\right](\theta) \sin \theta d \theta
$$

Finally, one deduces (30) by substituting in (B2) the above quantity $p_{b}^{1}$.

\section{REFERENCES}

${ }^{1}$ L. Pilon, "Foams in glass manufacture," in Foam engineering: Fundamentals and Aplications, edited by P. Stevenson (John Wiley \& Sons, Ltd, Chichester, UK, 2012) Chap. 16, pp. 355-409.

${ }^{2}$ S. H. Lee and L. G. Leal, "The motion of a sphere in the presence of a deformable interface. II. A numerical study of the translation of a sphere normal to an interface," J. Colloid Interface Sci. 87, 81-106 (1982). 
${ }^{3}$ C. Berdan and L. G. Leal, "Motion of a sphere in the presence of a deformation interface. I. Perturbation of the interface from flat: The effects on drag and torque," J. Colloid Interface Sci. 87, 62-80 (1982).

${ }^{4}$ S. G. Yiantsios and R. H. Davis, "On the buoyancy-driven motion of a drop towards a rigid surface or a deformable interface," J. Fluid Mech. 217, 547-573 (1990).

${ }^{5}$ G. K. Youngren and A. Acrivos, "Stokes flow past a particle of arbitrary shape: a numerical method of solution," J. Fluid Mech. 69, 377-403 (1975).

${ }^{6}$ S. Kim and S. J. Karrila, Microhydrodynamics. Principles and selected applications (Martinus Nijhoff Publishers, The Hague, 1983).

${ }^{7}$ C. Pozrikidis, Boundary integral and singularity methods for linearized viscous flow (Cambridge University Press, Cambridge, 1992).

${ }^{8}$ A. Sellier, "Boundary element technique for slow viscous flows about particles," in Boundary Element Methods in Engineering and Sciences, Vol. 4 (World Scientific, 2010) Chap. 7, pp. 239-281.

${ }^{9}$ H. Kočárková, F. Rouyer, and F. Pigeonneau, "Film drainage of viscous liquid on top of bare bubble: Influence of the bond number," Phys. Fluids 25, 022105 (2013).

${ }^{10}$ F. Pigeonneau and A. Sellier, "Low-Reynolds-Number gravity-driven migration and deformation of bubbles near a free surface," Phys. Fluids 23, 092102 (2011).

${ }^{11}$ G. Hetsroni and S. Haber, "The flow in and around a droplet or bubble submerged in an unbounded arbitrary velocity field," Rheol. Acta 9, 488-496 (1970).

${ }^{12}$ E. Chervenivanova and Z. Zapryanov, "On the deformation of two droplets in a quasisteady Stokes flow," Int. J. Multiphase Flow 11, 721-738 (1985).

${ }^{13}$ E. Chervenivanova and Z. Zapryanov, "The slow motion of droplets perpendicular to a deformable flat fluid interface," Q. J. Mech. Appl. Math. 41, 419-444 (1988).

${ }^{14} \mathrm{R}$. Aris, Vectors, Tensors and the basic equation of fluid mechanics (Dover Publications, Inc, New York, 1962).

${ }^{15}$ D. D. Joseph and Y. Y. Renardy, Fundamentals of two-fluid dynamics. Part I: Mathematical Theory and Applications (Springer, New York, 1993).

${ }^{16}$ As shown in ${ }^{32}$, the integration of $\boldsymbol{\nabla}_{S} \cdot \boldsymbol{n}$ over the closed surface $S_{0}$ gives no contribution.

$$
\int_{S_{1}} \boldsymbol{\sigma} \cdot \boldsymbol{n} d S_{1}-\rho_{l} \mathcal{V}_{b} \boldsymbol{g}=\mathbf{0}, \mathcal{V}_{b}=4 \pi a^{3} / 3
$$

Clearly, (B5) means that the flow $\left(\boldsymbol{u}, p+\rho_{l} \boldsymbol{g} \cdot \boldsymbol{x}+p_{a}\right)$ exerts on the bubble a zero force. 
${ }^{17}$ E. Bart, "The slow unsteady settling of a fluid sphere toward a flat fluid interface," Chem. Eng. Sci. 23, 193-210 (1968).

${ }^{18}$ M. Meyyappan, W. R. Wilcox, and R S. Subramanian, "Thermocapillary migration of a bubble normal to a plane surface," J. Colloid Interface Sci. 83, 199-208 (1981).

${ }^{19}$ J. Happel and H. Brenner, Low Reynolds number hydrodynamics (Martinus Nijhoff Publishers, The Hague, 1983).

${ }^{20}$ Misprints occurring in the Appendix of the reference ${ }^{18}$ have been however corrected when used in the present analysis.

${ }^{21}$ Z. Zapryanov and S. Tabakova, Dynamics of bubbles, drops and rigid particles (Kluwer academic publishers, Dordrecht, 1999).

${ }^{22} \mathrm{M}$. Guémas, Low-Reynolds-Number gravity-driven migration and deformation of bubble $(s)$ and/or solid particle(s) near a deformable free surface, Ph.D. thesis, École Polytechnique, université Paris-Saclay (2014).

${ }^{23}$ M. Abramowitz and I. A. Stegun, Handbook of mathematical functions (Dover Publications, Inc., New York, 1965).

${ }^{24}$ For $l=6 a$ it is found that $\lambda_{0, s} \sim 1.142$ and $\lambda_{0} \sim 1.091$. Since Bo/ Ca $\sim 1.67$ one has $\lambda_{0} \neq \mathrm{Bo} / \mathrm{Ca}$, i. e. the bubble is not force free in that case.

${ }^{25}$ Those conditions at the poles are not clearly required in ${ }^{13}$.

${ }^{26}$ L. Landau and E. Lifchitz, Mécanique des fluides (Mir, Moscou, 1994).

${ }^{27}$ M. Guémas, A. Sellier, and F. Pigeonneau, "Slow viscous gravity-driven interaction between a bubble and a free surface with unequal surface tensions," Phys. Fluids 27, 043102 (2015).

${ }^{28}$ A. Sellier, "Thermocapillary motion of a two-bubble cluster near a plane solid wall," Comptes Rendus Mécanique 333, 8, 636-641 (2005).

${ }^{29}$ L. Pasol and A. Sellier, "Migration of a solid particle in the vicinity of a plane fluid-fluid interface," Eur. J. Mech. B-Fluid 30, 76-88 (2011).

${ }^{30}$ I. S. Gradshteyn and I. M. Ryzbik, Table of integrals, series, and products (Academic Press, San Diego, 1965).

${ }^{31}$ M. Stimson and G. B. Jeffery, "The motion of two spheres in a viscous fluid," Prod. R. Soc. London, serie A 111, 110-116 (1926).

${ }^{32}$ L. G. Leal, Advanced transport phenomena: Fluid mechanics and convective transport processes (Cambridge University Press, New York, 2007). 